Conditions Contributing to Adverse Loading of Wind Turbines in the Nocturnal Boundary Layer: Final Report

November 15, 2003 - December 31, 2004

D.C. Fritts

Colorado Research Associates

Boulder, Colorado
Subcontract Report NREL/SR-500-37809 July 2005 


\section{Conditions Contributing to}

Adverse Loading of Wind Turbines in the Nocturnal Boundary Layer: Final Report

November 15, 2003 - December 31, 2004

D.C. Fritts

Colorado Research Associates

Boulder, Colorado

NREL Technical Monitor: Neil Kelley

Prepared under Subcontract No. LEE-4-33232-01 


\section{NOTICE}

This report was prepared as an account of work sponsored by an agency of the United States government. Neither the United States government nor any agency thereof, nor any of their employees, makes any warranty, express or implied, or assumes any legal liability or responsibility for the accuracy, completeness, or usefulness of any information, apparatus, product, or process disclosed, or represents that its use would not infringe privately owned rights. Reference herein to any specific commercial product, process, or service by trade name, trademark, manufacturer, or otherwise does not necessarily constitute or imply its endorsement, recommendation, or favoring by the United States government or any agency thereof. The views and opinions of authors expressed herein do not necessarily state or reflect those of the United States government or any agency thereof.

Available electronically at http://www.osti.gov/bridge

Available for a processing fee to U.S. Department of Energy and its contractors, in paper, from:

U.S. Department of Energy

Office of Scientific and Technical Information

P.O. Box 62

Oak Ridge, TN 37831-0062

phone: 865.576 .8401

fax: 865.576 .5728

email: mailto:reports@adonis.osti.gov

Available for sale to the public, in paper, from:

U.S. Department of Commerce

National Technical Information Service

5285 Port Royal Road

Springfield, VA 22161

phone: 800.553 .6847

fax: 703.605.6900

email: orders@ntis.fedworld.gov

online ordering: http://www.ntis.gov/ordering.htm

This publication received minimal editorial review at NREL 


\section{CONTENTS}

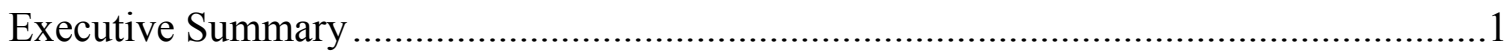

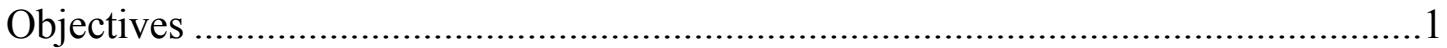

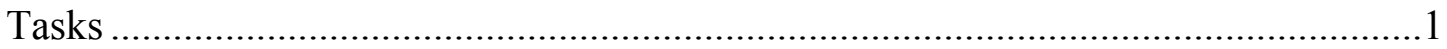

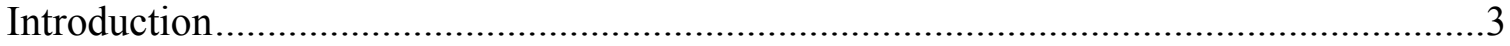

Field Data Analysis Methodology .......................................................................4

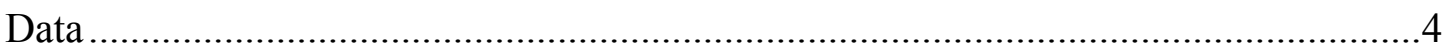

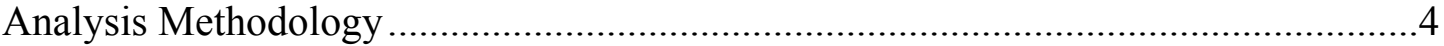

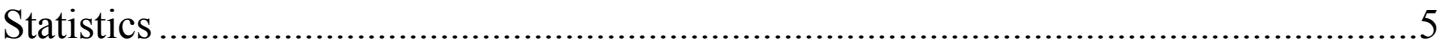

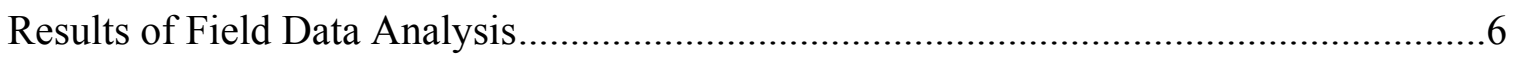

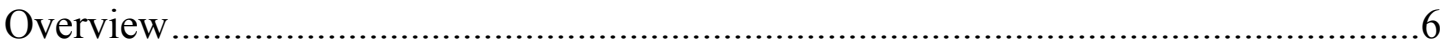

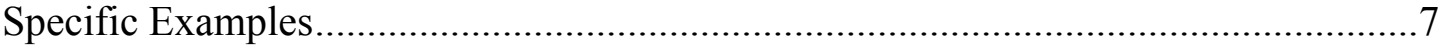

Focused Interval From Anemometer Array .......................................................27

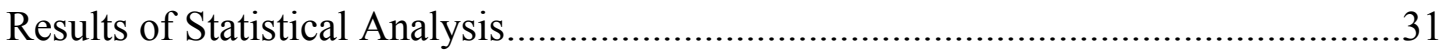

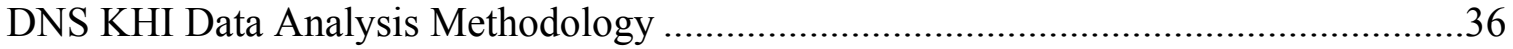

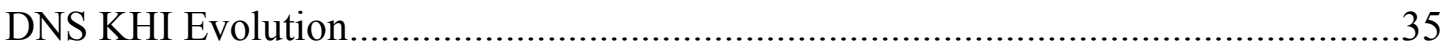

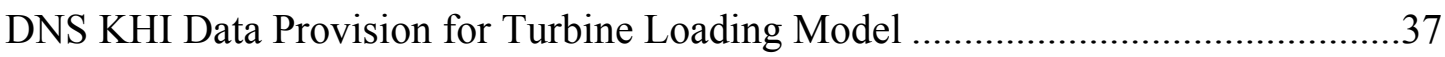

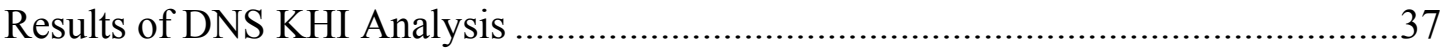

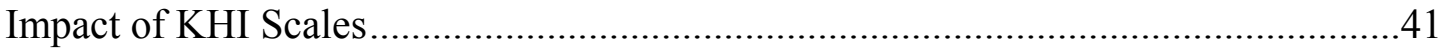

Recommendations for Implementing an Adverse Loading Risk Warning......................41

Conditions for Significant Turbulence.............................................................4

Recommended Measurements and Analysis .........................................................42

Predicting Boundary Layer Flows and Mesocale Models ....................................43

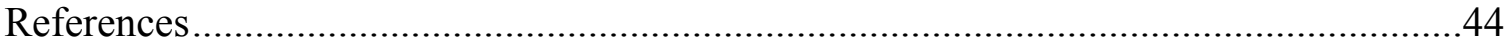




\section{FIGURES}

Figure 1. Data from 00001100 UT for September 1........................................... 8 - 10

Figure 2. As in Figure 1 for data collected on September 2................................12 - 15

Figure 3. As in Figure 1 for data collected on September 3 ..................................16 - 19

Figure 4. As in Figure 1 for data collected on September $10-14$ at $67 \mathrm{~m} \square$

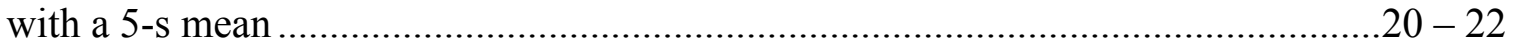

Figure 5. Horizontal winds at 54 and $116 \mathrm{~m}$ averaged for $30 \mathrm{~s}$.................................23

Figure 6. As in Figure 4b, but expanding the data..............................................24-26

Figure 7. Component velocities for September 13, 2003 from 0745 to 0755 UT

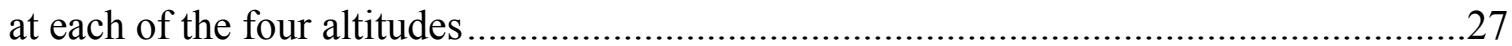

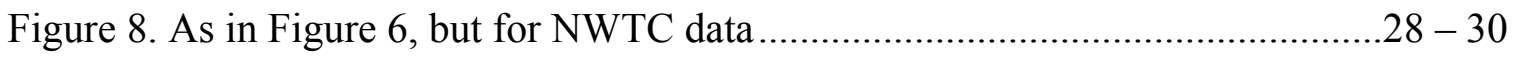

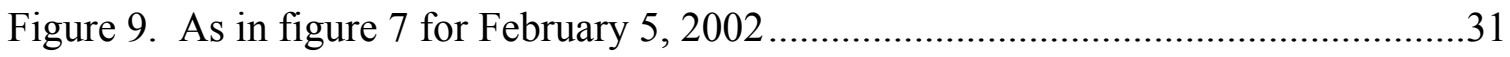

Figure 10. Volumetric views of instability and turbulence caused by KHI.....................36

Figure 11. Temperature (left) and velocity (right) variance profiles ..............................38

Figure 12. Compensated (i.e., divided by $r^{2}$ ) second-order structure functions ................39

Figure 13. Profiles of structure-function fit parameters................................................39

Figure 14. Streamwise and spanwise averages ................................................... 40

\section{TABLES}

Table 1. Number of days when the maximum number of points with vorticity magnitude

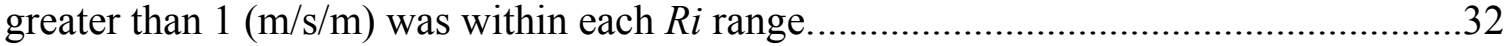
Table 2. Total component vorticity means (upper left value), standard deviations (upper right value), and number of points (lower value) contributing for each CTKE range (top

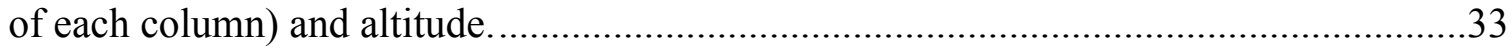

Table 3. As in Table 2, but for vertical velocity magnitude. ........................................34 


\section{Executive Summary}

Wind turbines represent an important emerging technology for power generation. However, their operations and benefits are often adversely affected, both in duty cycle and maintenance costs, by structures in the atmospheric flow field that contribute to excessive blade loading, stresses, vibrations, and mechanical fatigue or failure.

The features that create adverse turbine blade responses seem to be relatively small-scale vortex structures that arise naturally in turbulence (or its dynamical precursors) in atmospheric flows at high Reynolds numbers $(R e)$. Such features are often small in cross section but coherent along their length, and often have much more intense shears (or vorticity) than the flow at larger spatial scales. Larger scale flow features may also contribute to blade forcing, but because their local gradients are usually less significant, they may not contain the same potential for strong perturbations.

\section{Objectives}

The objectives of this work were to:

- Develop a methodology to describe the characteristics of coherent turbulence in the nocturnal atmospheric boundary layer that induce excessive structural loads and component vibration in operating wind turbines.

- Apply the results to develop techniques of real-time detection and prediction that can be used to mitigate the effects of turbulence.

\section{Tasks}

Before we initiated this effort for the National Renewable Energy Laboratory (NREL), we:

- Provided consultation services about the analysis and interpretation of data collected at or near the $120-\mathrm{m}$ General Electric wind meteorological tower in southeastern Colorado.

- Analyzed select NREL-furnished databases to assess turbulence conditions and sources that are likely to product adverse responses in operating wind turbines.

- Suggested key atmospheric parameters that may be applied in real time to warn coherent turbulent activity.

- Used mesoscale numerical models to identify the larger scale conditions that are involved in the evolution of coherent turbulence activity. These can guide the development of a predictive capability. 
- Provided recommendations for implementing a predictive capability of adverse turbulence activity based on normally available observations and any local supplemental information that would improve accuracy and reliability. 


\section{Introduction}

Wind turbines represent an important emerging technology for power generation. However, their operations and benefits are often adversely affected, in both duty cycle and maintenance costs, by structures in the atmospheric flow field that contribute to excessive blade loading, stresses, vibrations, and mechanical fatigue or failure.

The features that create adverse turbine blade responses seem to be relatively small-scale vortex structures that arise naturally in turbulence (or its dynamical precursors) in atmospheric flows at high Reynolds numbers $(R e)$. Such features are often small in cross section but coherent along their length, and often have much more intense shears (or vorticity) than the flow at larger spatial scales. Larger scale flow features may also contribute to blade forcing, but because their local gradients are usually less significant, they may not contain the same potential for strong perturbations.

To address these issues, we performed extensive analyses of atmospheric data obtained under stably stratified conditions in the nocturnal boundary layer (NBL) and assessments of wind shears, vortex structures, turbulent kinetic energy (TKE), coherent TKE (CTKE), and their correlations that arise from direct numerical simulations (DNS) of KelvinHelmholtz instability (KHI) at the spatial scales expected to be most important in turbine blade loading and stresses.

We used data collected in the NBL during the 1999 Cooperative Atmosphere-Surface Exchange Study (CASES-99) field program in Kansas (Poulos, et al. 2002) to develop analysis methods, which we applied to tower data that were collected at Lamar and provided by the National Renewable Energy Laboratory (NREL). The objective of the CASES-99 experiment performed in October 1999 was to study the dynamics of the day/night transitions of the nocturnal atmospheric boundary layer. The NREL analysis efforts focused on defining small-scale coherent features that could provide strong loading and stresses on turbine blades, their correlations with other dynamical variables (especially $C T K E$ ), and the environments in which they occurred. We sought to:

- Define the morphology and orientation of coherent vortex structures throughout a KHI event.

- Quantify small-scale vortex intensities as functions of Kelvin-Helmholz (KH) billow size.

- Assess correlations among turbulence quantities.

- Provide the results of a representative simulation for use in the NREL turbine windloading model.

We used high-resolution DNS of KHI performed for other purposes (Werne and Fritts 1998, 1999a, 1999b, 2000, 2001; Fritts and Werne 2000; Werne et al. 2001).

Our specific methodologies and results are described in the following sections. 


\section{Field Data Analysis Methodology}

\section{Data}

Two primary data sets were used in this analysis. The first was provided by NREL and consisted of tower observations made from September 1-15, 2003 near Lamar, Colorado. The tower instruments recorded temperature and three-dimensional (3-D) winds at $20 \mathrm{~Hz}$ at 54,67, 85, and $116 \mathrm{~m}$. The second was from a tower at the CASES-99 field experiment from October 5-29, 1999. We observed 3-D winds at $20 \mathrm{~Hz}$ at $1.5,5,10,20,30,40,50$, and $55 \mathrm{~m}$, and thermocouple temperatures at $5 \mathrm{~Hz}$ at 0.23 and $0.67 \mathrm{~m}$, then every $1.8 \mathrm{~m}$ to $58.1 \mathrm{~m}$.

We obtained a third, very brief, high-resolution data set (duration $\sim 11 \mathrm{~min}$ ) on February 5, 2001 from the National Wind Technology Center (NWTC). It had different instrument altitudes than did the longer Lamar 2003 data set, which was also analyzed because it offered a unique opportunity to examine the forcing derived from simultaneous inflow measurements and the corresponding structural response of an operating wind turbine undergoing a severe loading event.

\section{Analysis Methodology}

Wind and temperature data were collected from each vertical level from 00-11 UTC $\mathrm{UT}^{\S}$ to observe the overnight evolution and short-term variability of the NBL. For the Lamar data, winds and temperatures were available at each level. For CASES-99 data, winds at each level were used, and temperatures were calculated at those levels by interpolating from the thermocouples directly above and below. We used the data at each Lamar and CASES-99 wind sensor to calculate the cross-stream vorticity components and the CTKE. The Richardson number $(R i)$ was calculated based on the vertical gradients of filtered horizontal winds and temperatures between each pair of wind measurements. Filtering was performed by averaging a 5-s window of data, stepping every $5 \mathrm{~s}$. A 5 -s averaging was employed to provide sensitivity to those times when the mean flow varied rapidly. (Estimates of TKE and CTKE depend on the averaging interval because a longer average allows for a greater instantaneous departure from the mean for all perturbation quantities). Thus, a 30 -s mean and a $4 \mathrm{~m} / \mathrm{s}$ mean wind imply coherent structures as large as $120 \mathrm{~m}$ may contribute to the perturbations, whereas a 5 -s mean and the same $4 \mathrm{~m} / \mathrm{s}$ mean wind enable only a $20-\mathrm{m}$ structure to contribute to perturbation quantities.) We used a 0.1-s window and step and applied a shorter filter to reduce uncertainties for higher frequency wind estimates.

Spacings for the Ri computations at Lamar were 13, 18, and $31 \mathrm{~m}$. For CASES-99 these spacings were $3.5,5,10,10,10,10$, and $5 \mathrm{~m}$ with increasing altitude. $R i$ for each layer was then estimated from the mean temperature, temperature gradient, and vector horizontal wind shear derived from the 30-s data with the expression

\footnotetext{
${ }^{\S}$ UTC or UT refers to Universal Coordinated Time and is 7 hours earlier than the local time (Mountain Standard Time) at the Lamar Site; i.e., 0820 UT is 0120 MST locally.
} 


$$
R i=(g / T)\left(d T / d z+g / c_{p}\right) /\left((d u / d z)^{2}+(d v / d z)^{2}\right)
$$

where $g$ is the acceleration due to gravity, $T$ is the thermodyamic or potential temperature, $z$ is the height above the ground, $c_{p}$ is the specific heat of air at constant presure, and $u$ and $v$ are the mean zonal (E-W) and meridional (N-S) wind components.

Cross-stream vorticity components were derived for each level where wind data were available. The two components that could be assessed are $d u_{\text {perp }} / d x$ and $d w / d x$, where $u_{\text {perp }}$ and $w$ are the 0.1-s horizontal perpendicular wind (based on departures from the 30-s horizontal wind, $U_{o}$ ) and vertical wind and the $x$ direction is along the 30-s horizontal wind. Spatial derivatives (and cross-stream vorticities) were estimated by assuming that advection dominated local temporal evolution as follows:

$$
d u_{\text {perp }} / d x=\left(d u_{\text {perp }} / d t\right) / U_{o}
$$

and

$$
d w / d x=(d w / d t) / U_{o}
$$

The turbulent kinetic energy TKE was calculated from the differences between the filtered and high frequency winds (0.1-s winds minus the 30-s winds, defined as $u^{\prime}, v^{\prime}$, and $w^{\prime}$ ) as

$$
T K E=\left(u^{2}+v^{\prime 2}+w^{2}\right) / 2=\left(u_{p a r}{ }^{2}+u_{p e r p}{ }^{2}+w^{2}\right) / 2
$$

The coherent turbulent kinetic energy CTKE was calculated from the same terms as

$$
C T K E=\left[\left(u^{\prime} v^{\prime}\right)^{2}+\left(u^{\prime} w^{\prime}\right)^{2}+\left(v^{\prime} w^{\prime}\right)^{2}\right]^{1 / 2} / 2
$$

\section{Statistics}

Statistics were compiled for the entire Lamar and CASES-99 data sets. When the magnitude of either cross-stream vorticity component was greater than 1 (units of $\mathrm{m} / \mathrm{s} / \mathrm{m}$, or a change of the magnitude of that component by more than $1 \mathrm{~m} / \mathrm{s}$ across a $1-\mathrm{m}$ distance), the values of $R i$ and $C T K E$ were recorded and stored. Most of the time, the $C T K E$ was less than $10 \mathrm{~m}^{2} / \mathrm{s}^{2}$, so we define this to be a nominal threshold for significance. To assess the significance of large CTKE, we also computed the means of the total component vorticity $\left[\left(\left(d u_{\text {perp }} / d x\right)^{2}+(d w / d x)^{2}\right)^{1 / 2}\right]$ and $w$ for each of several ranges of CTKE, 1-2, 2-5, 5-10, and $>10$, above the nominal threshold for significant values. Results of this analysis are discussed in the next section. 


\section{Results of Field Data Analysis}

\section{Overview}

Data from each field program exhibited significant variability and intermittency of stability states, larger scale wave motions and instabilities, and smaller scale turbulence throughout each night. Several general statements can be made about turbulence (and larger scale coherent motions) as indicated in the velocity data and by our estimates of component vorticity, CTKE, or TKE (not displayed, as the correlation with CTKE was very high in an averaged sense; see DNS discussion).

1. $\square$ Turbulence events vary in duration from $\sim 1 \mathrm{~min}$ to several hours.

2. $\square$ Turbulence motions are almost always correlated in the velocity and vorticity fields, and to a slightly lesser degree between these fields and either CTKE or TKE.

3. $\square$ Turbulence can appear and disappear quickly, probably because of advection (transport by the velocity field) rather than temporal evolution.

4. $\square$ Stronger turbulence events are usually extended in altitude and exhibit similar temporal variability at multiple altitudes. However, turbulence frequently occurs at lower altitudes, but is more intermittent or absent at higher altitudes. In these cases, $R i$ at upper levels is at least $1 / 2$ and often greater than 1 .

5. $\square$ Significant levels of turbulence occur for $R i$ well above and below $1 / 4$. Often, however, sustained large component vorticities and CTKE appear to correlate with $R i$ near zero or weakly positive.

6. $\square$ Significant $C T K E$ typically occurs during turbulence events, which is apparent in the velocity or vorticity component data. This correlation increases with altitude.

7. $\square$ Turbulence onsets or bursts are difficult to relate to specific flow features, partly because spatially localized features are advected.

8. $\square$ Three classes of flows appear to have a high predictive value. One includes frontal passages (and microfronts) that lead to rapid changes in mean wind of a few $\mathrm{m} / \mathrm{s}$ over a few minutes.

9. $\square$ Enhanced turbulence often accompanies larger scale oscillations (wave motions) that have periods of a few to 30 minutes and enhanced turbulence in the upward phase.

10. Turbulence is also enhanced, and $\sqsubset$ more continuous, when it accompanies more obvious instability dynamics that have significant amplitudes and shorter periods, typically a few to tens of seconds. 
11. The largest values of CTKE and of vertical velocity are not caused by strong turbulence; rather, they result from larger scale coherent oscillatory motions with periods of 5-30 s.

12. Large oscillatory motions with large CTKE, large vertical motions, and large component vorticities (which often include turbulence) are nearly always correlated with significant mean wind shears and small $R i$, and appear to be the major hazards to wind turbines.

\section{Specific Examples}

\section{General Character of Turbulence and Correlations}

The temporal variability, correlations among computed quantities, and turbulence (or lack thereof) at high and low $R i$ are illustrated well with the component winds, component vorticities, CTKE, and Ri obtained from Lamar data on September 1 (see Figure 1). The four panels reveal each quantity computed at $54,67,85$, and $116 \mathrm{~m}$, with $R i$ computed above each layer, except at the highest level. Of note are:

- The sudden variations in turbulence intensity (as described by CTKE)

- A general correlation in occurrence at all altitudes

- A lack of correlation of turbulence onset or cessation with any obvious features in the mean wind

- A correlation in nominal (but not peak) values between vorticity components and CTKE. 
Lamar Tower: 1 Sep 2003: Ri Layer $=85-116 \mathrm{~m}$. Vorticity layer $=116 \mathrm{~m}$

Ri Filtering $=5 \mathrm{~s}$ ave every $5 \mathrm{~s}$. Vorticity filtering $=0.10 \mathrm{~s}$ ave every $0.10 \mathrm{~s}$.

Average velocity $=5.11 \mathrm{~m} / \mathrm{s}$.
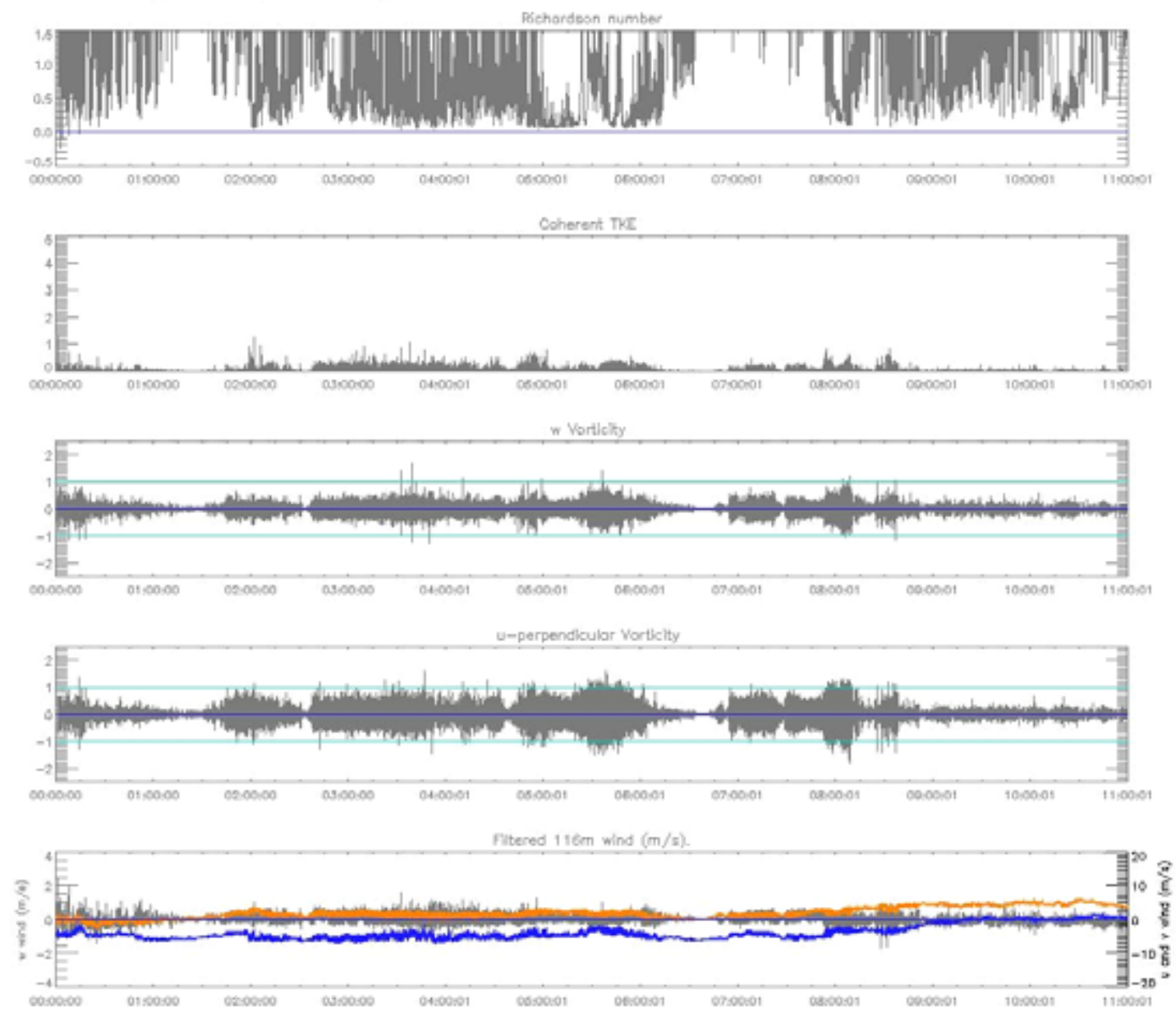

Figure 1a 
Lamar Tower: 1 Sep 2003: Ri Layer $=67-85 \mathrm{~m}$. Vorticity layer $=67 \mathrm{~m}$

Ri Filtering $=5 \mathrm{~s}$ ave every $5 \mathrm{~s}$. Vorticity filtering $=0.10 \mathrm{~s}$ ave every $0.10 \mathrm{~s}$.

Average velocity $=4.64 \mathrm{~m} / \mathrm{s}$.
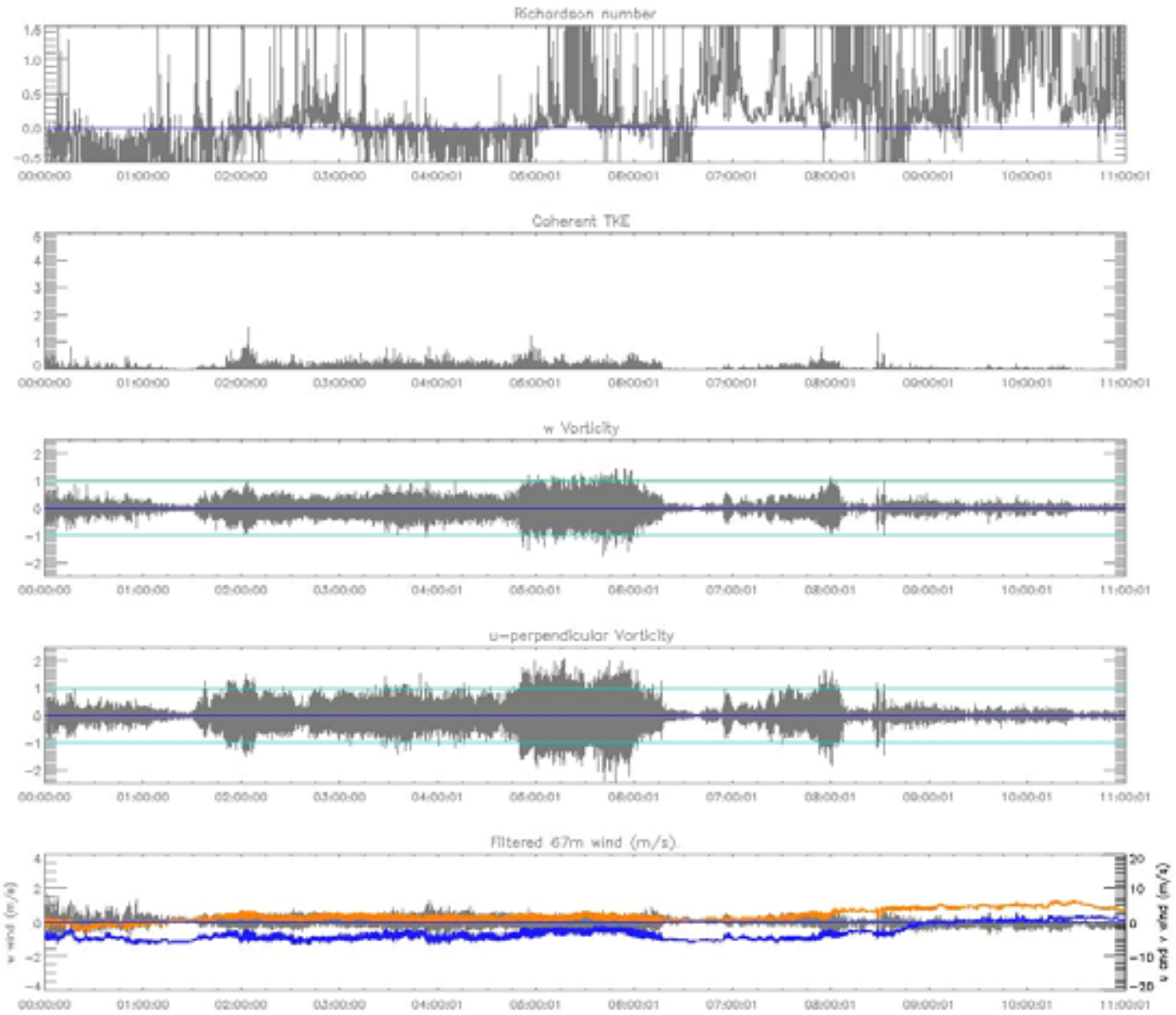

Figure 1b 
Lamar Tower: 1 Sep 2003: Ri Layer $=85-116 \mathrm{~m}$. Vorticity layer $=85 \mathrm{~m}$

Ri Filtering $=5$ s ave every $5 \mathrm{~s}$. Vorticity filtering $=0.10 \mathrm{~s}$ ave every $0.10 \mathrm{~s}$.

Average velocity $=4.86 \mathrm{~m} / \mathrm{s}$.
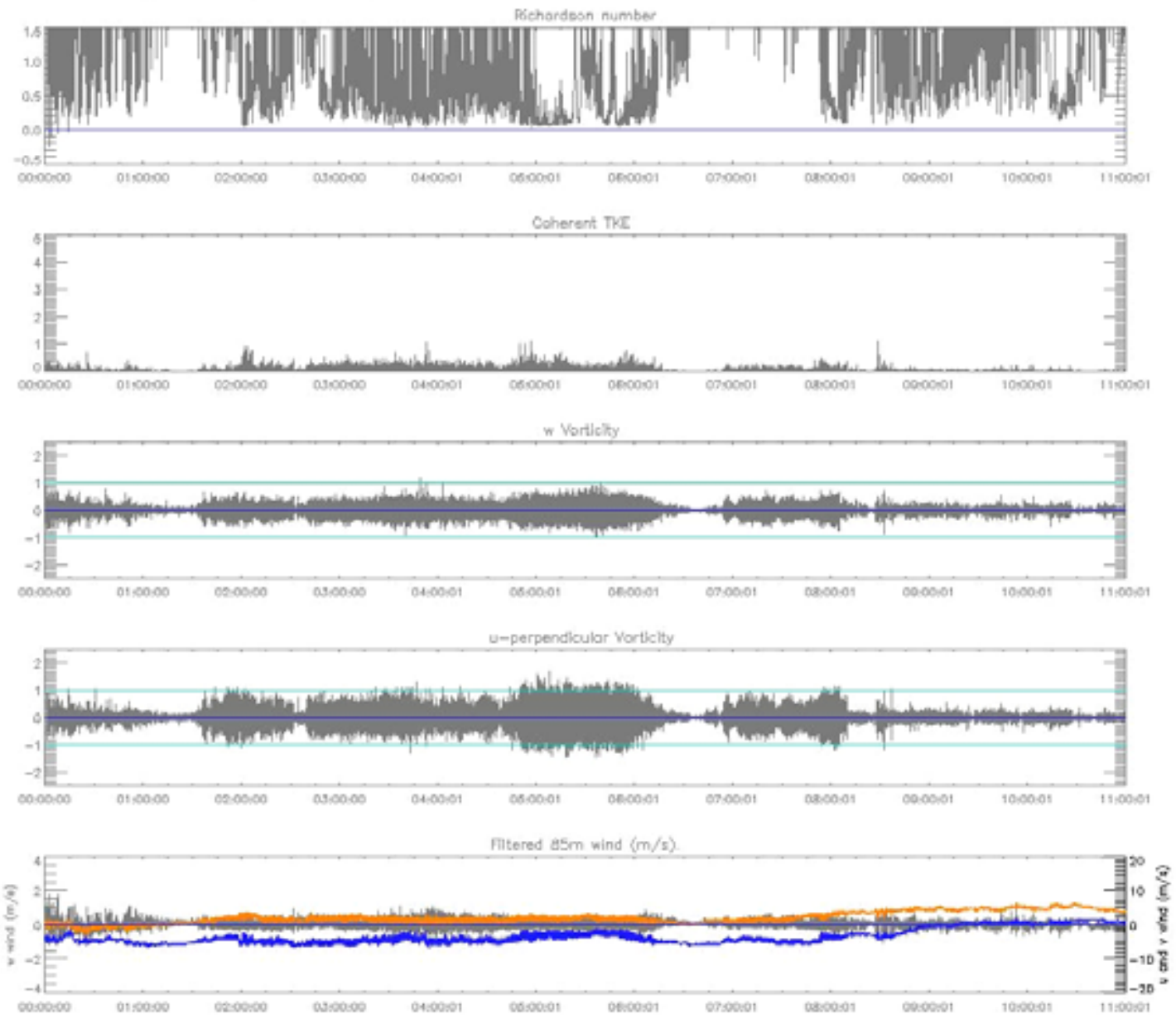

Figure 1c

Figure 1. Data from 00001100 UT for September 1. Panels show data for sensors at 54 (1a), 67 (1b), 85 (1c), and $116 \mathrm{~m}$ (1d) altitudes (top to bottom). Within each panel are displayed Ri, CTKE, vertical and horizontal perpendicular vorticity, and the component velocities ( $u$ blue, $v$ orange, $w$ gray) (top to bottom) based on a 30-s mean wind. $R i$ in each case is for the layer above, except at the highest level, where $R i$ for the layer below is displayed.

\section{Exceptions to the General Behavior}

In addition to the general character, Figure 1 indicates several intervals with $R i<0$ and weak turbulence (see panels $\mathrm{a}$ and $\mathrm{b}$ of Figure 1 at $\sim 0120$ UT) and others with sustained $R i \sim 1 / 2$ or larger and significant turbulence (see especially panels $\mathrm{c}$ and $\mathrm{d}$ of Figure 1 at early and late times). Turbulence can arise at small spatial scales where the local $R i$ is small, but that are not captured by $R i$ estimates over more extended depths. 
Variations of turbulence in altitude are illustrated with data obtained at Lamar on September 2 and 3, 2003 (Figures 2 and 3). In these cases, turbulence is nearly continuous at $54 \mathrm{~m}$, but becomes intermittent or absent at higher altitudes. This appears to be associated in each case with a larger increase in mean $R i$ at higher altitudes than seen in Figure 1. Figures 2 and 3 also show significantly higher values of CTKE, but comparable values of component vorticities. There are also hints that the large CTKE values at higher altitudes occur at minima in $R i$ that may not be adequately resolved. As noted earlier, it is not easy to relate appearance or disappearance of turbulence with specific variations in the mean wind. There are examples of:

- Turbulence cessation with increasing $R i$ (see the higher altitudes at $0820 \mathrm{UT}$ on September 1, near 0400 UT on September 2, and near 0300 and 0800 UT on September 3)

- Turbulence appearance at high $R i$ (see the higher altitudes at 0700 UT on September 1) and turbulence cessation as $R i$ falls below zero (see the lower altitudes at 0615 UT on September 1). 
Lamar Tower: 2 Sep 2003: Ri Layer $=54-67 \mathrm{~m}$. Vorticity layer $=54 \mathrm{~m}$

Ri Filtering $=5 \mathrm{~s}$ ave every $5 \mathrm{~s}$. Vorticity filtering $=0.10 \mathrm{~s}$ ave every $0.10 \mathrm{~s}$.

Average velocity $=7.33 \mathrm{~m} / \mathrm{s}$.
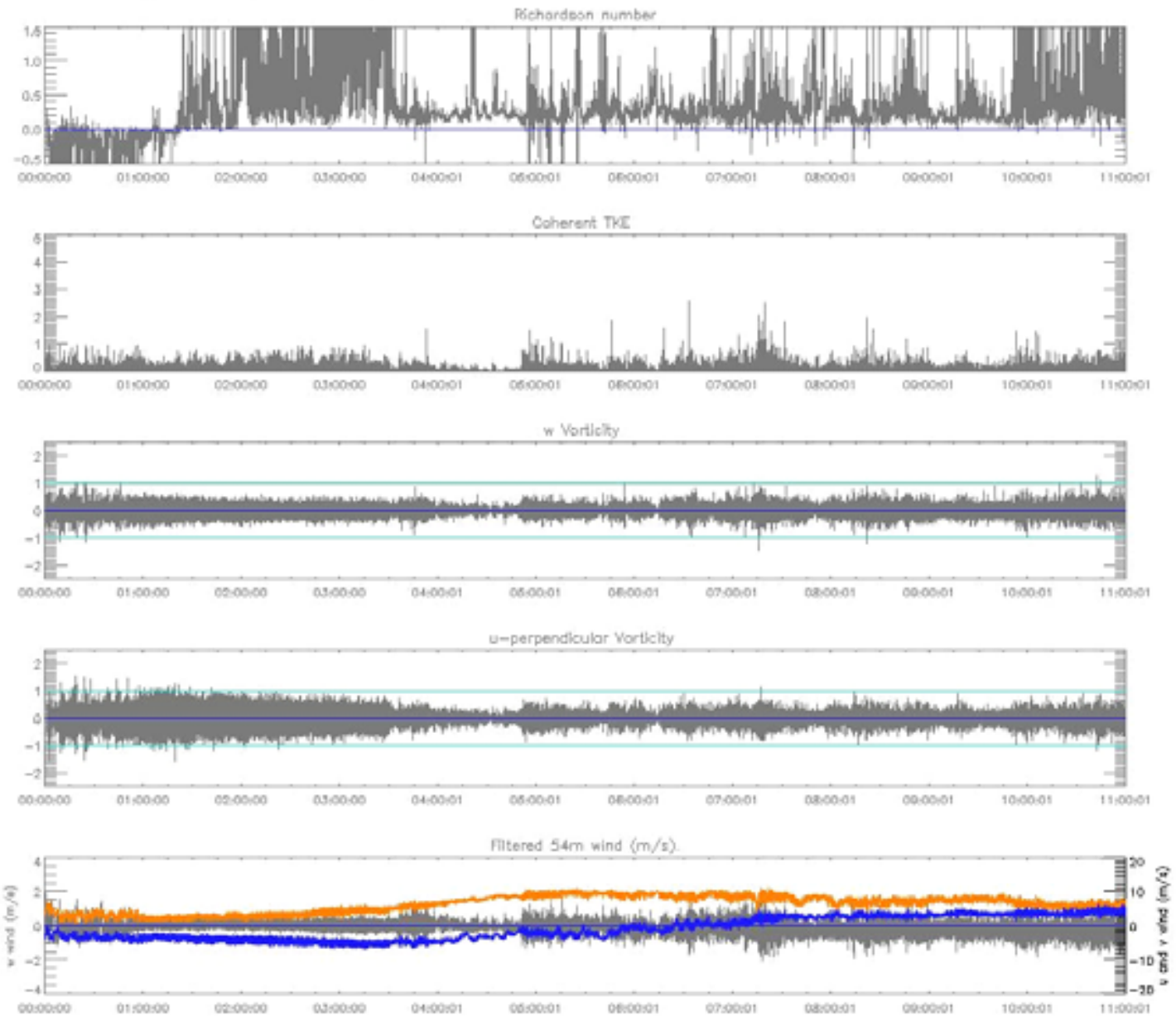

Figure 2a 
Lamar Tower: 2 Sep 2003: Ri Layer= 67-85m. Vorticity layer= $67 \mathrm{~m}$

Ri Filtering $=5 \mathrm{~s}$ ave every $5 \mathrm{~s}$. Vorticity filtering $=0.10 \mathrm{~s}$ ave every $0.10 \mathrm{~s}$.

Average velocity $=7.74 \mathrm{~m} / \mathrm{s}$
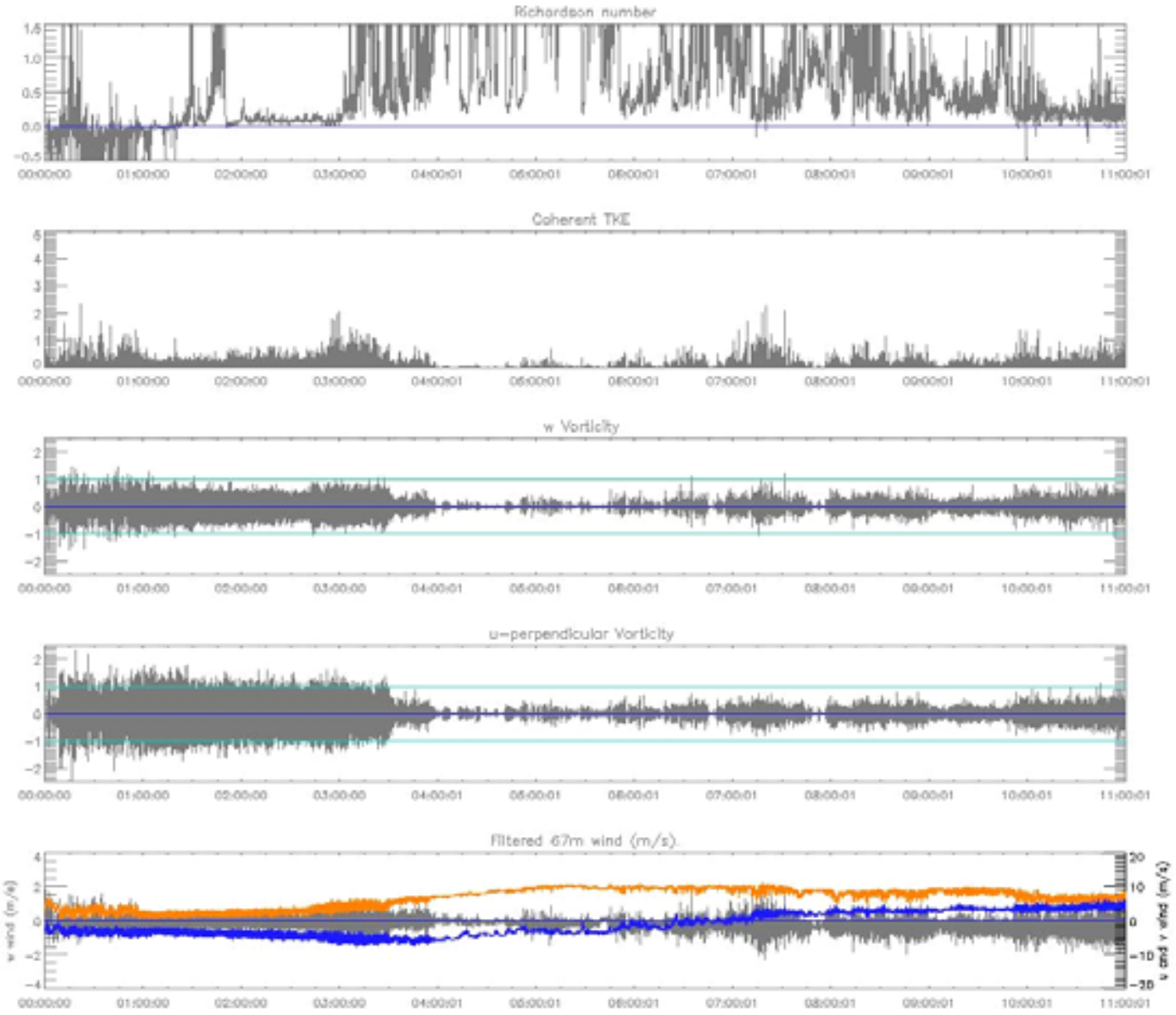

Figure 2b 
Lamar Tower: 2 Sep 2003: Ri Layer $=85-116 \mathrm{~m}$. Vorticity layer $=85 \mathrm{~m}$

Ri Filtering $=5 \mathrm{~s}$ ave every $5 \mathrm{~s}$. Vorticity filtering $=0.10 \mathrm{~s}$ ave every $0.10 \mathrm{~s}$.

Average velocity $=8.35 \mathrm{~m} / \mathrm{s}$
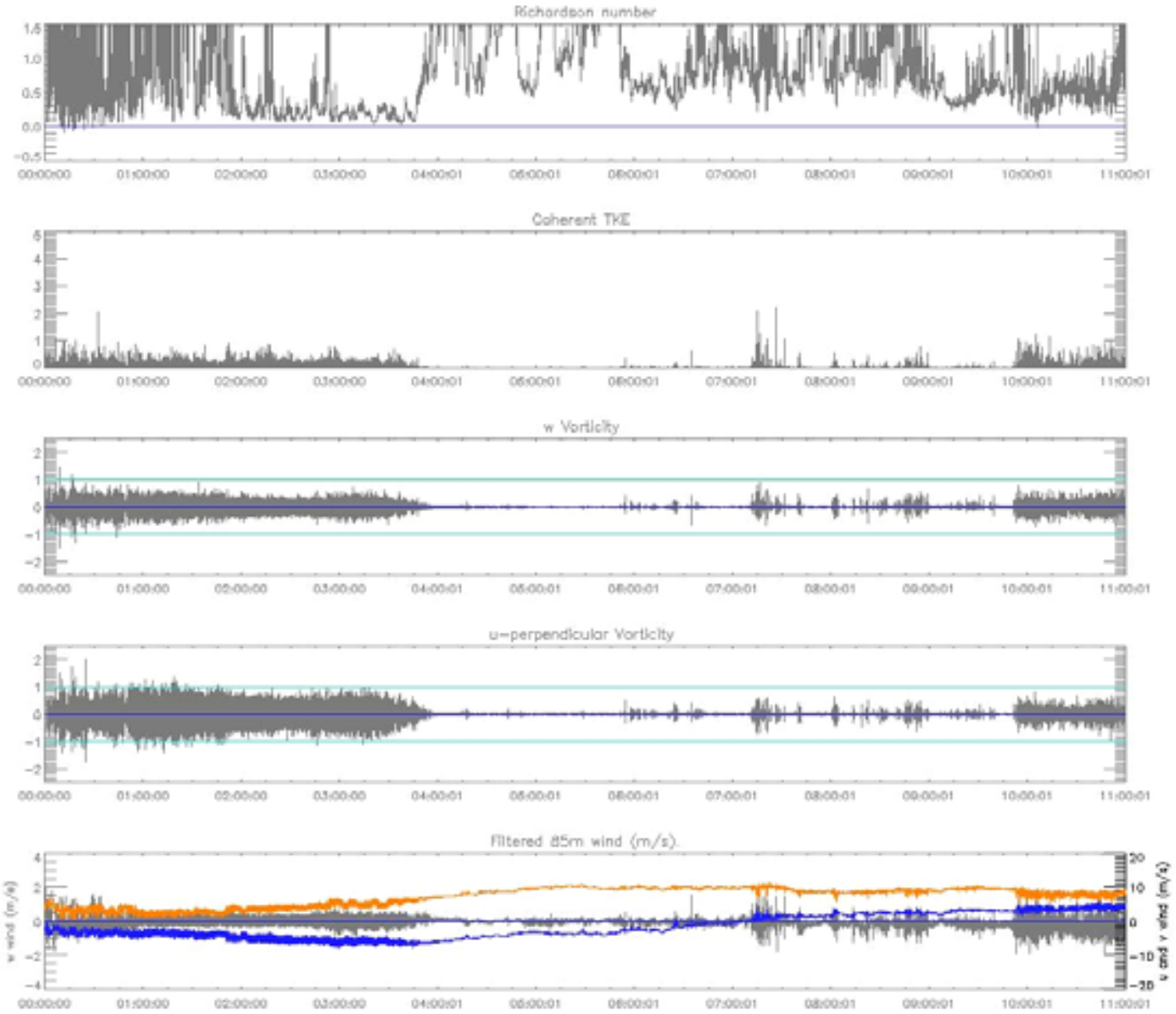

Figure 2c 

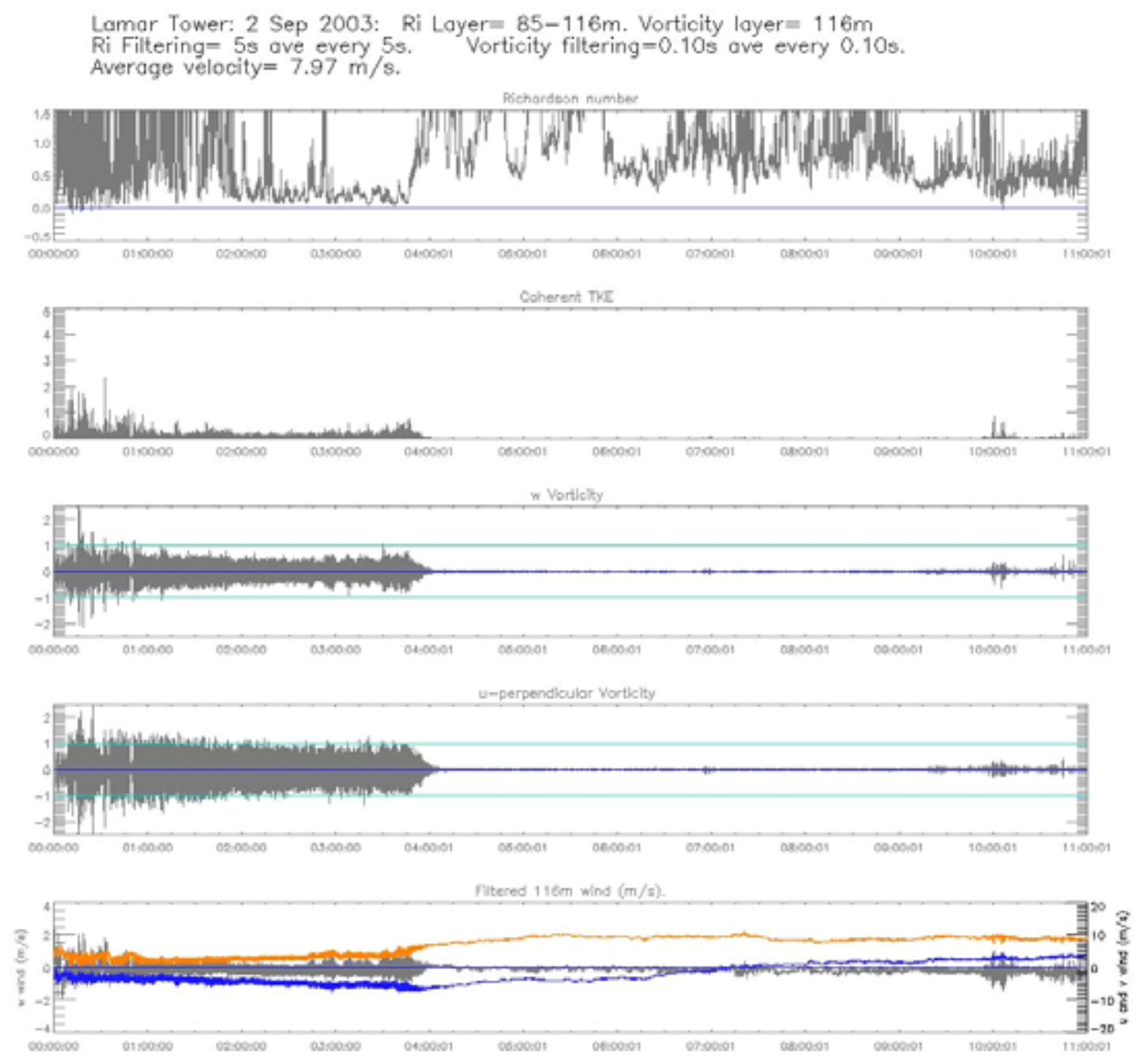

Figure 2d. As in Figure 1 for data collected on September 2. 
Lamar Tower: 3 Sep 2003: Ri Layer $=54-67 \mathrm{~m}$. Vorticity layer $=54 \mathrm{~m}$

Ri Filtering $=5 \mathrm{~s}$ ave every $5 \mathrm{~s}$. Vorticity filtering $=0.10 \mathrm{~s}$ ave every $0.10 \mathrm{~s}$.

Average velocity $=8.34 \mathrm{~m} / \mathrm{s}$.
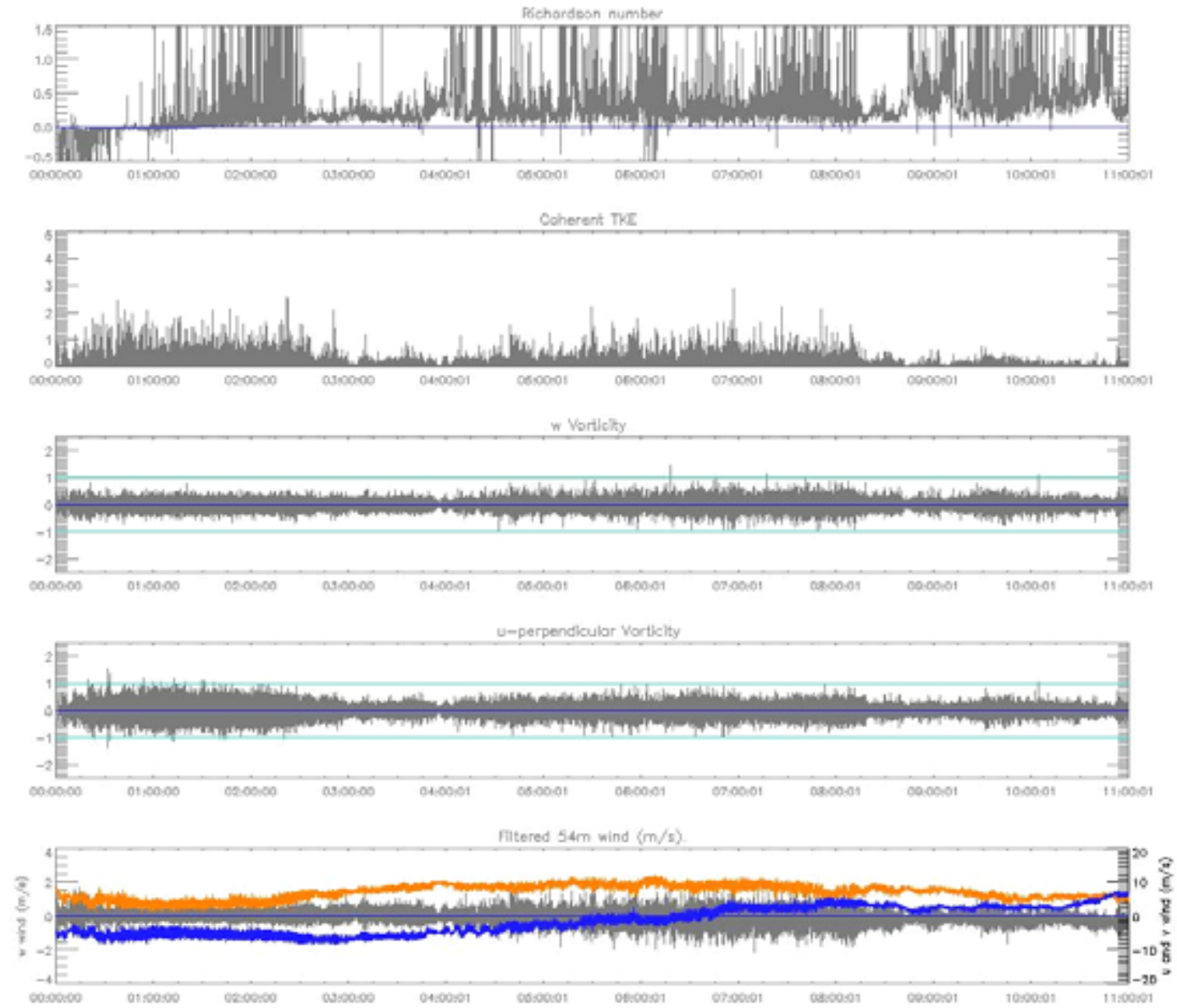

Figure 3a 
Lamar Tower: 3 Sep 2003: Ri Layer $=67-85 \mathrm{~m}$. Vorticity layer $=67 \mathrm{~m}$

Ri Filtering $=5 s$ ave every $5 \mathrm{~s}$. Vorticity filtering $=0.10 \mathrm{~s}$ ave every $0.10 \mathrm{~s}$.

Average velocity $=8.81 \mathrm{~m} / \mathrm{s}$
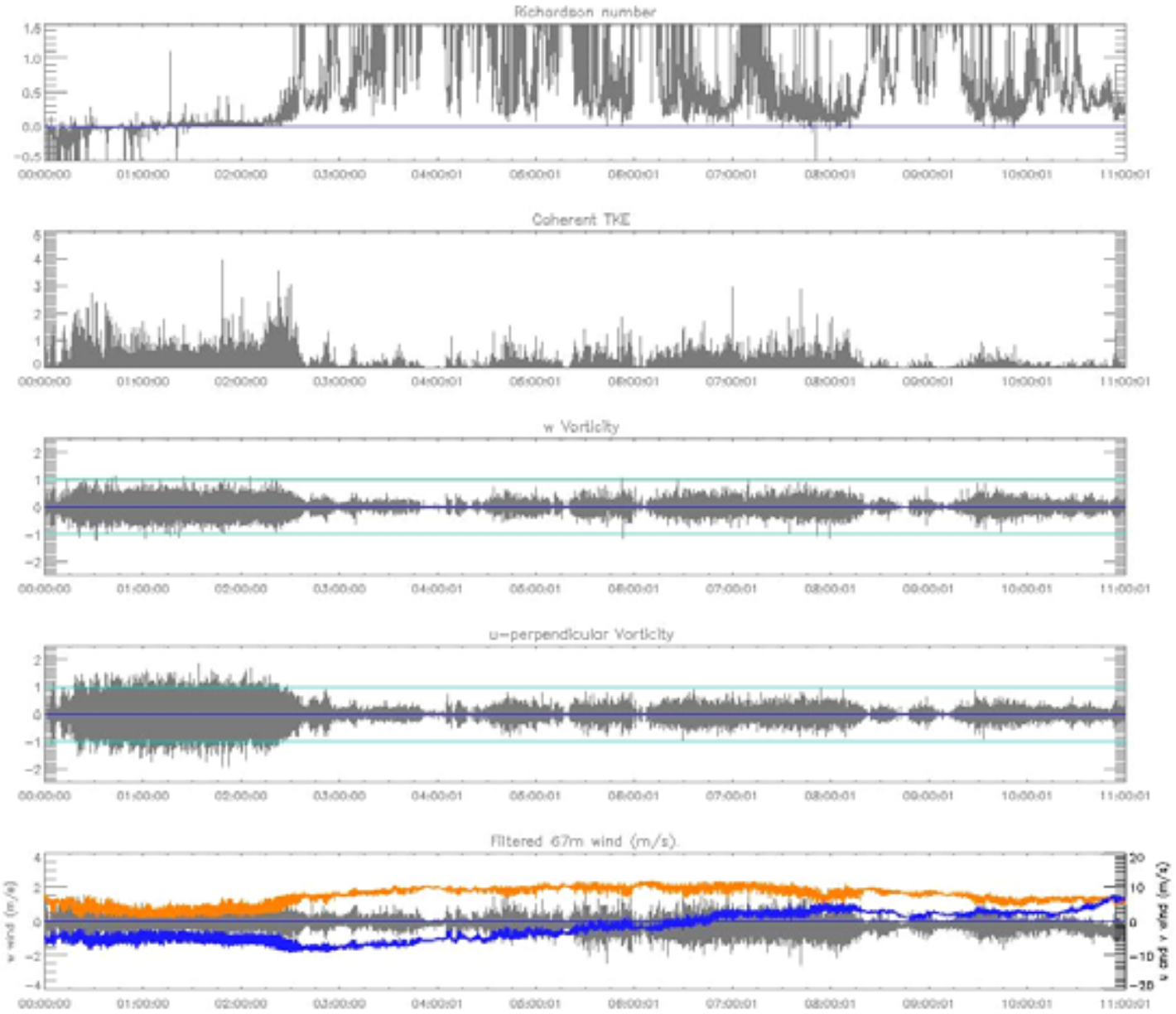

Figure 3b 
Lamar Tower: 3 Sep 2003: Ri Layer $=85-116 \mathrm{~m}$. Vorticity layer $=85 \mathrm{~m}$

Ri Filtering $=5 \mathrm{~s}$ ave every $5 \mathrm{~s}$. Vorticity filtering $=0.10 \mathrm{~s}$ ave every $0.10 \mathrm{~s}$.

Average velocity $=9.52 \mathrm{~m} / \mathrm{s}$
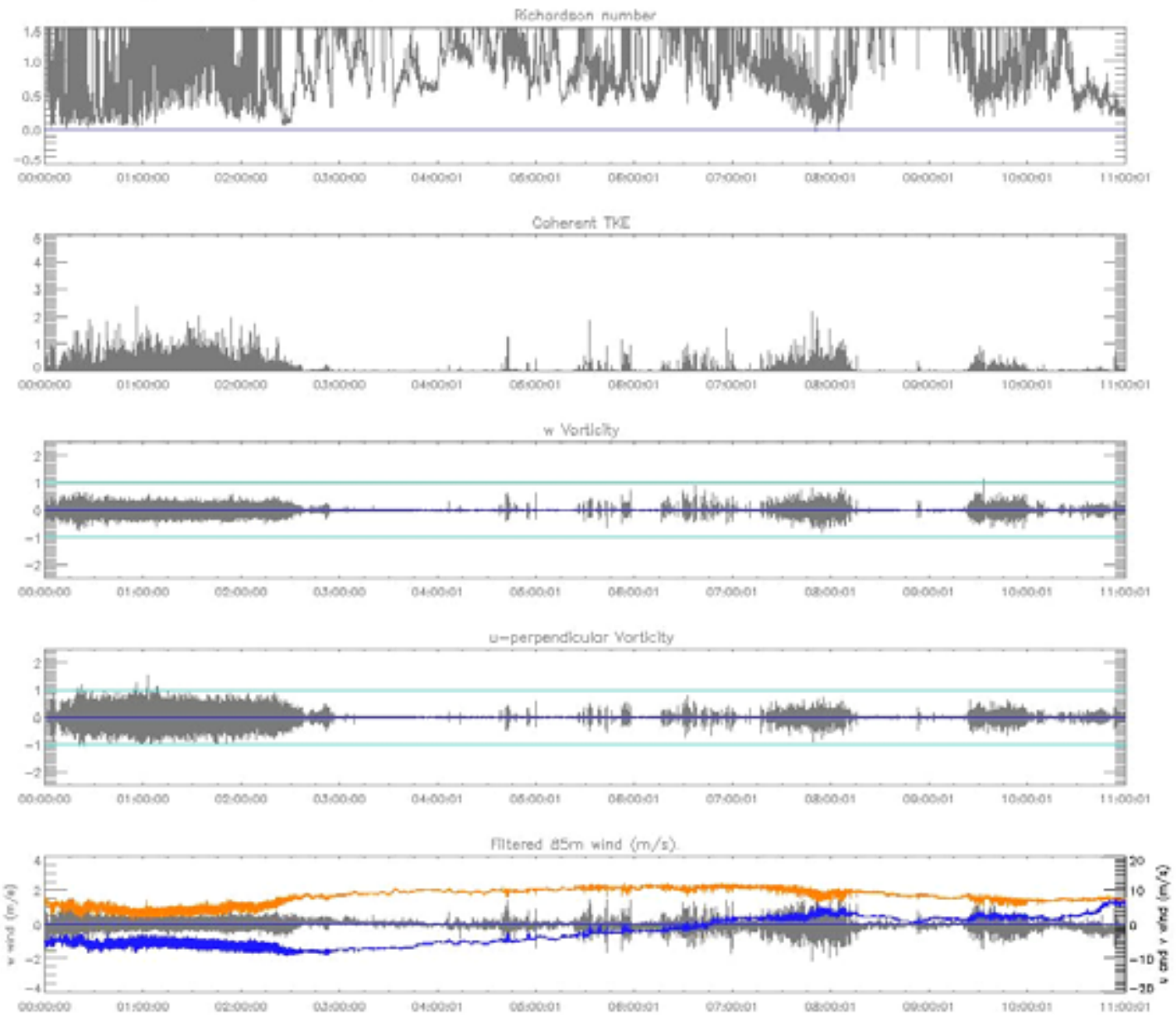

Figure 3c 
Lamar Tower: 3 Sep 2003: Ri Layer= $85-116 \mathrm{~m}$. Vorticity layer= $116 \mathrm{~m}$

Ri Filtering $=5 \mathrm{~s}$ ave every $5 \mathrm{~s}$. Vorticity filtering $=0.10 \mathrm{~s}$ ave every $0.10 \mathrm{~s}$.

Average velocity $=9.24 \mathrm{~m} / \mathrm{s}$
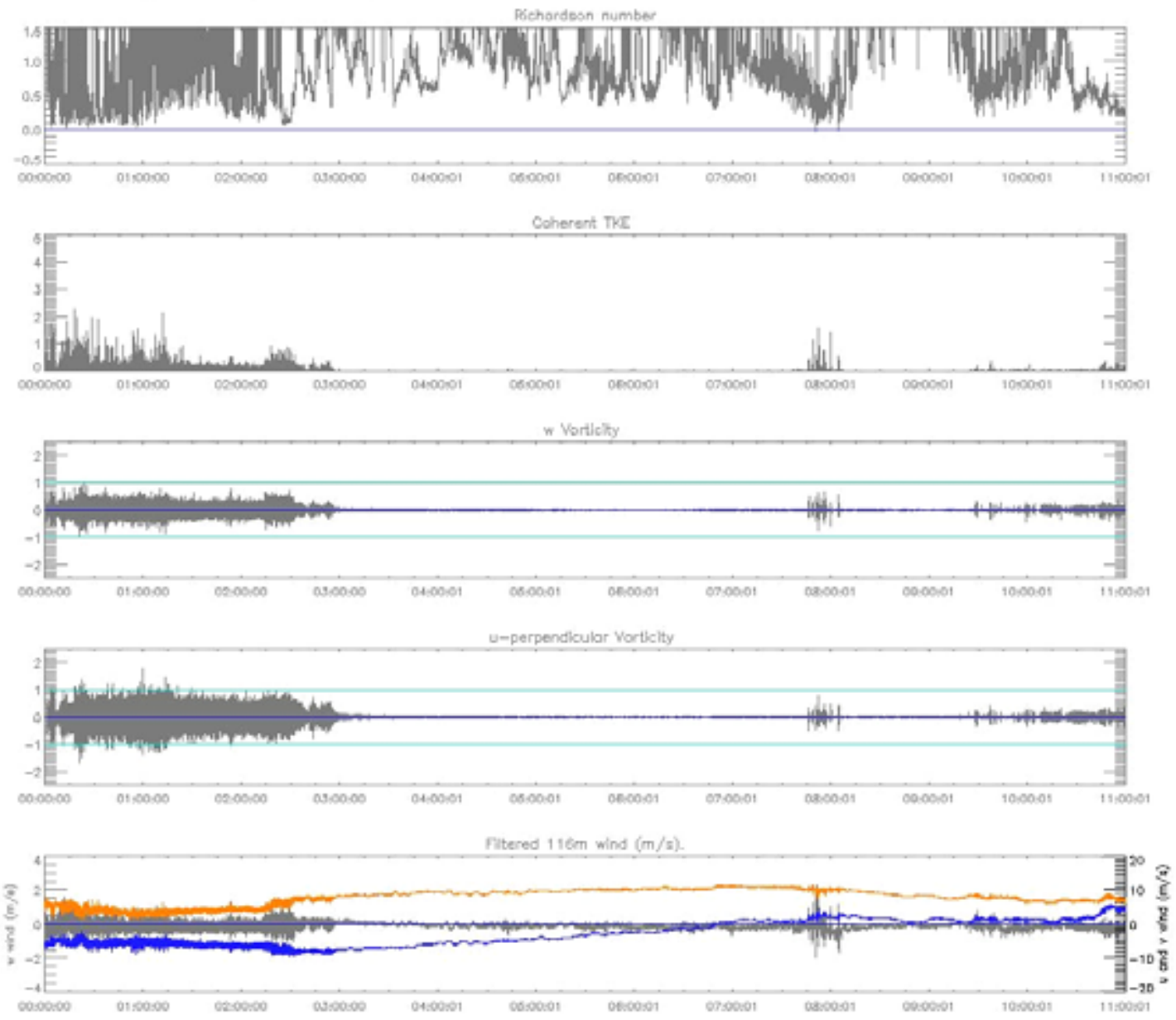

Figure 3d. As in Figure 1 for data collected on September 3.

\section{Turbulence Enhancements That Accompany Fronts, Waves, and Instabilities}

Several specific flow features seem to be associated with turbulence onset or enhancement. The most obvious in our analysis are frontal passages, or even microfronts, that exhibit relatively sudden changes in wind direction or magnitude of $2-20 \mathrm{~m} / \mathrm{s}$ or greater. Such events in Lamar are seen at $\sim 0130$ UT on September 10, 0745 UT on September 13, and $\sim 0230$ and 0620 UT on September 14 (see Figure 4). These typically have associated reductions of $R i$ to near or below zero and large increases in velocity variances, component vorticities, and CTKE that may last several hours. 
Lamar Tower: 10 Sep 2003: Ri Layer $=67-85 \mathrm{~m}$. Vorticity layer $=67 \mathrm{~m}$

Ri Filtering $=5 \mathrm{~s}$ ave every $5 \mathrm{~s}$. Vorticity filtering $=0.10 \mathrm{~s}$ ave every $0.10 \mathrm{~s}$.

Average velocity $=10.82 \mathrm{~m} / \mathrm{s}$.

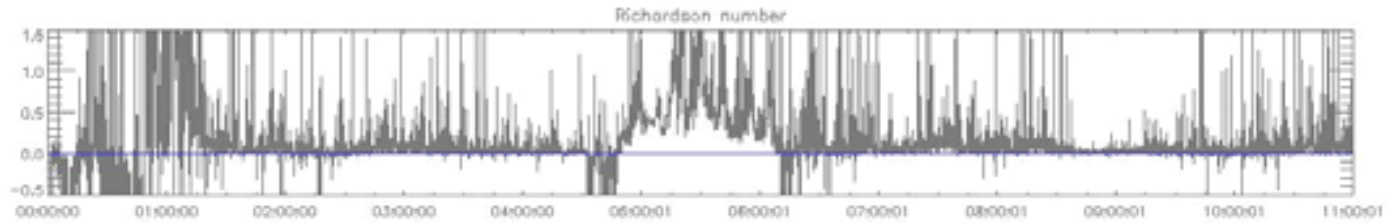

Doherent TKC

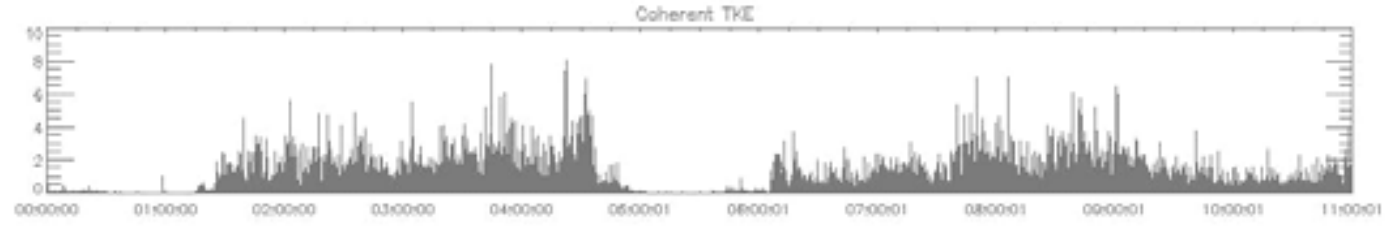

worticky
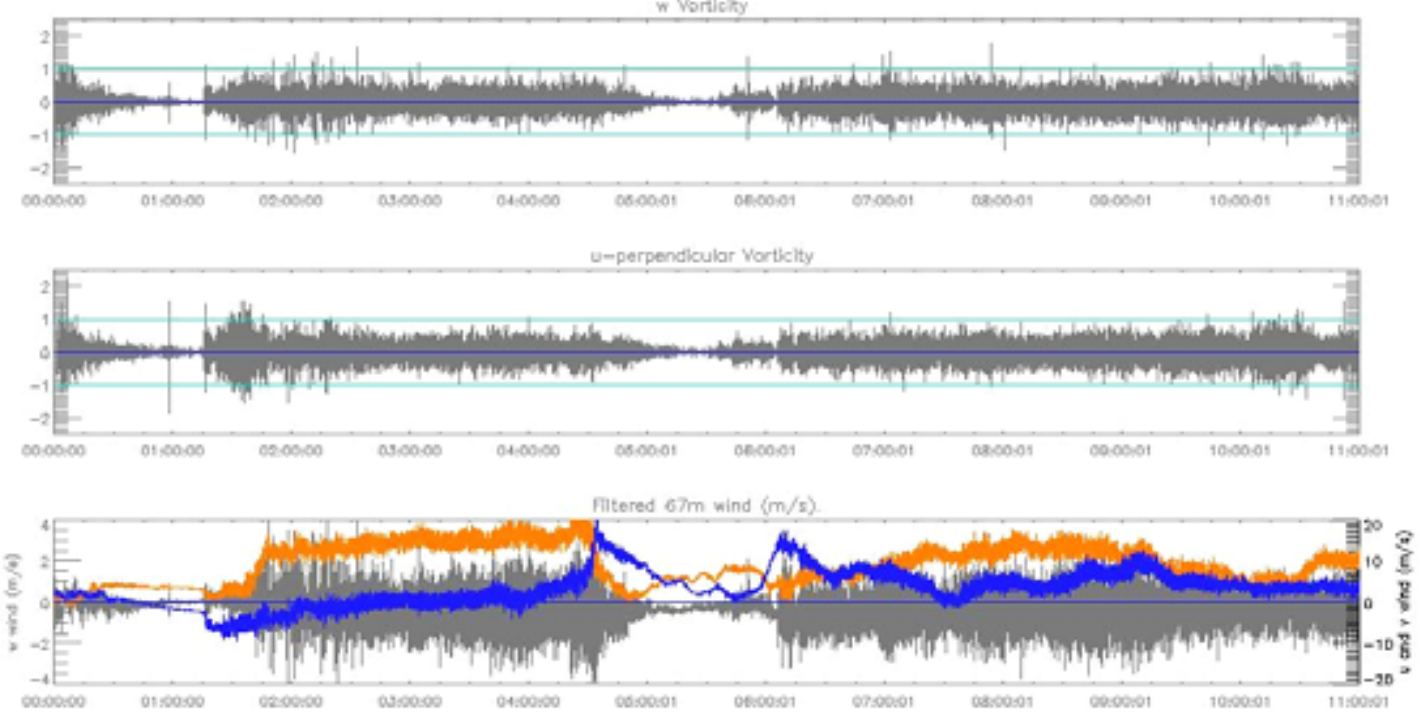

Figure 4a. As in Figure 1 for data collected on September 10 at $67 \mathrm{~m}$ with a 5 -s mean. 
Lamar Tower: 13 Sep 2003: Ri Layer $=67-85 \mathrm{~m}$. Vorticity layer= $67 \mathrm{~m}$

Ri Filtering $=5 \mathrm{~s}$ ave every $5 \mathrm{~s}$. Vorticity filtering $=0.10 \mathrm{~s}$ ave every $0.10 \mathrm{~s}$.

Average velocity $=11.56 \mathrm{~m} / \mathrm{s}$
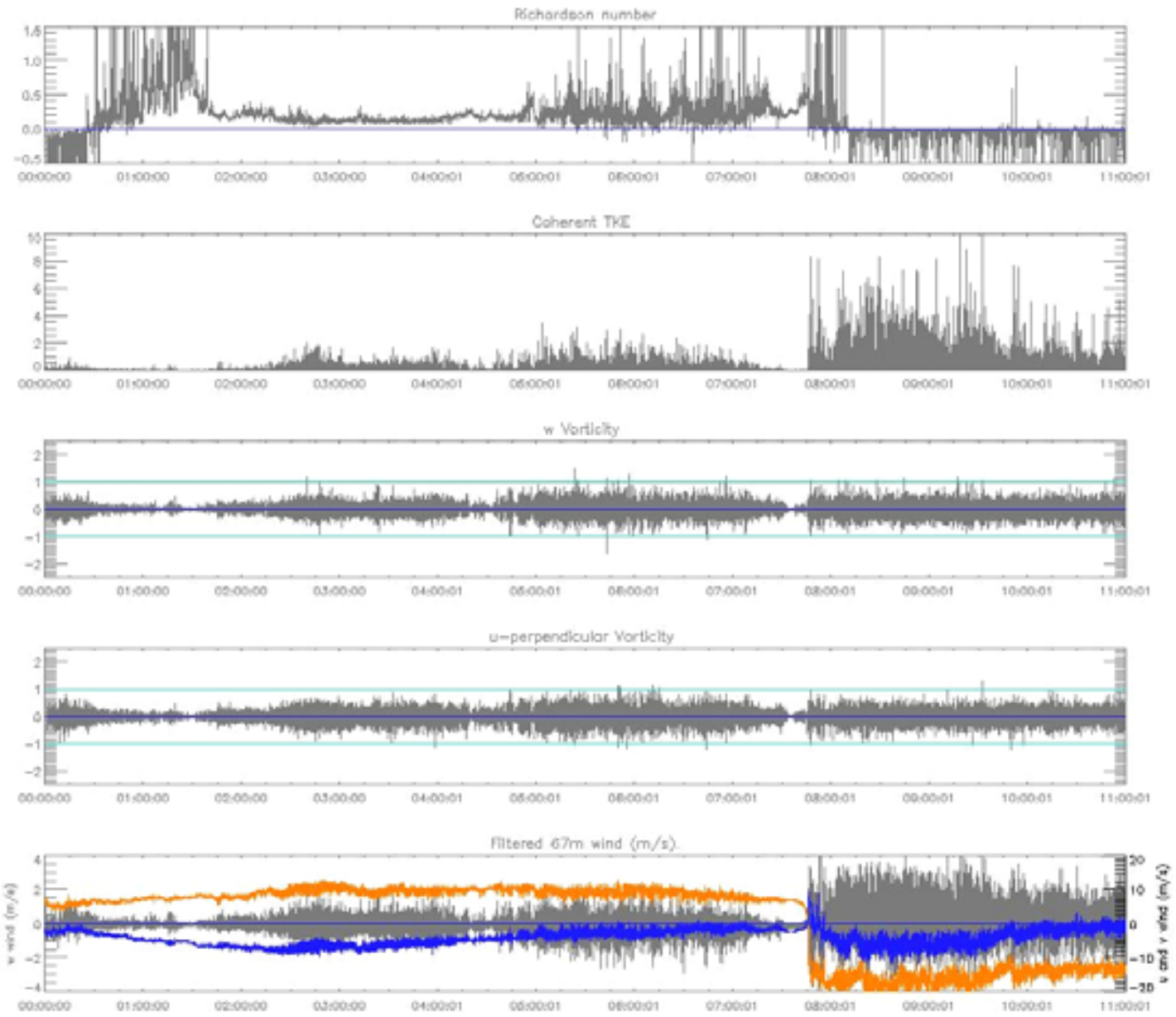

Figure 4b. As in Figure 1 for data collected on September 13 at $67 \mathrm{~m}$ with a 5 -s mean. 
Lamar Tower: 14 Sep 2003: Ri Layer $=67-85 \mathrm{~m}$. Vorticity layer $=67 \mathrm{~m}$

Ri Filtering $=5 s$ ave every $5 \mathrm{~s}$. Vorticity filtering $=0.10 \mathrm{~s}$ ave every $0.10 \mathrm{~s}$.

Average velocity $=7.26 \mathrm{~m} / \mathrm{s}$

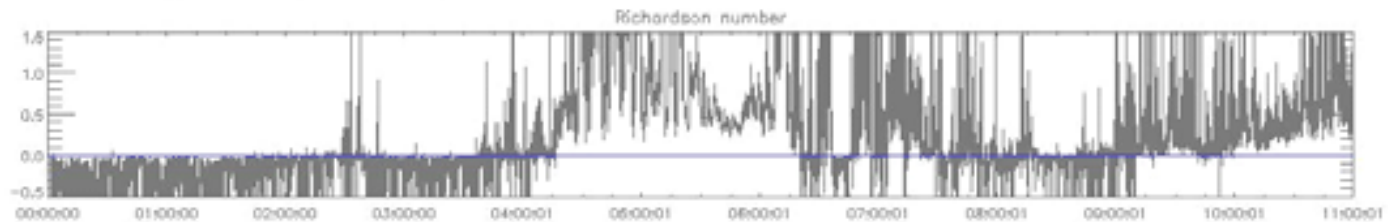

Coherent TKC
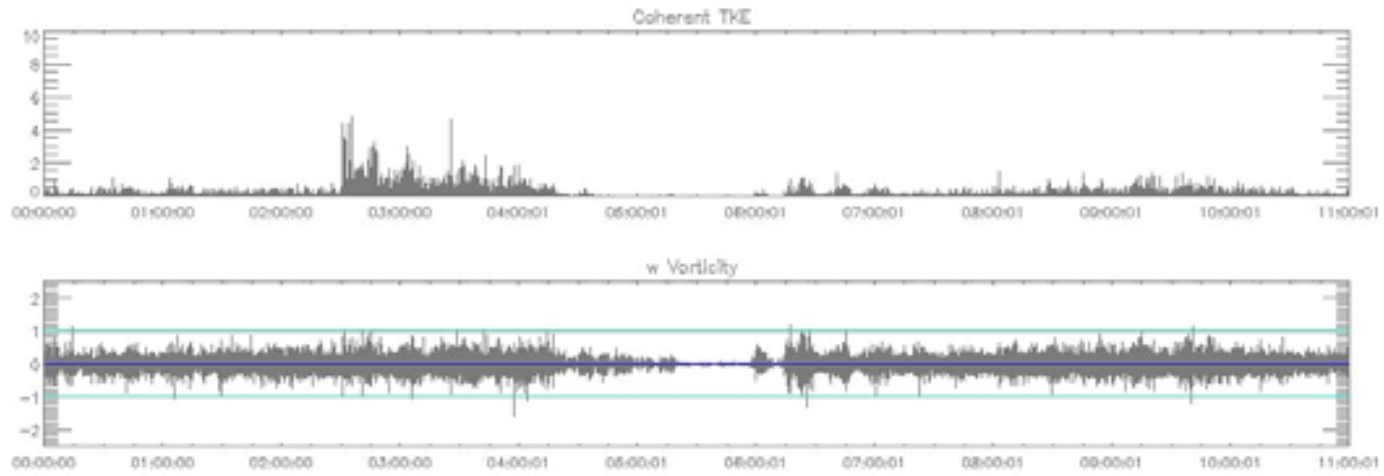

u-perpendicular Worticity

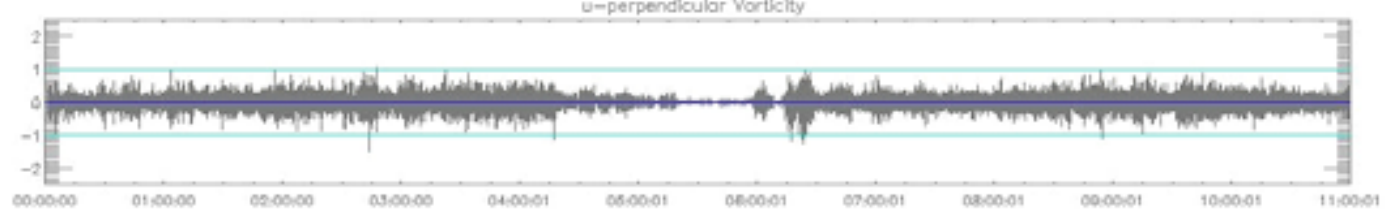

Fitered $67 \mathrm{~m}$ wind $\langle\mathrm{m} / \mathrm{s}$ )

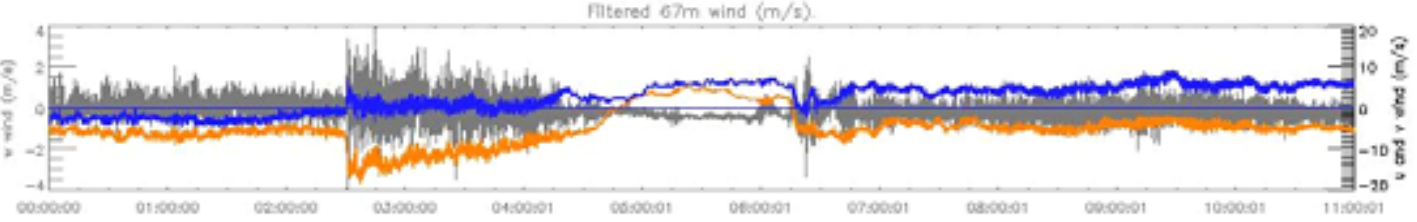

Figure 4c. As in Figure 1 for data collected on September 14 at $67 \mathrm{~m}$ with a 5-s mean.

Other apparent triggers for turbulence appear to be wave and instability processes. Internal gravity waves favor local instability development at the upward phase of the wave motion in direct numerical simulations and atmospheric observations. Instabilities at smaller scales, whether caused by larger scale wave motions, mean shears, or a combination of the two, provide a direct pathway to TKE. An indication of the mean structure necessary for significant turbulence is provided by horizontal winds averaged for $30 \mathrm{~s}$ at 54 and $116 \mathrm{~m}$ for September 10 (see the upper two panels of Figure 5). Comparison of these data with those shown in Figure $4 \mathrm{a}$ demonstrates that large fluctuating velocities, component vorticities, and CTKE (and TKE) correlate strongly with significant shears in the mean winds. Indeed, this event contains easily the largest vertical velocities (well above $10 \mathrm{~m} / \mathrm{s}$ ) and sustained high component vorticities and $C T K E$ of the entire Lamar data set (apart from that discussed below). Large shears by themselves, however, do not imply instability and turbulence, as demonstrated by the interval from 0500-0600 UT on September 10 (see Figures 4a and 5), where significant shears do not lead to instability and turbulence because $R i$ is large. 
Lamar Tower: 10 Sep 2003: Ri Layer= 85-116m. Vorticity layer= $116 \mathrm{~m}$

Ri Filtering $=30 \mathrm{~s}$ ove every $30 \mathrm{~s}$. Vorticity filtering $=0.10 \mathrm{~s}$ ave every $0.10 \mathrm{~s}$.

Average velocity $=12.84 \mathrm{~m} / \mathrm{s}$.
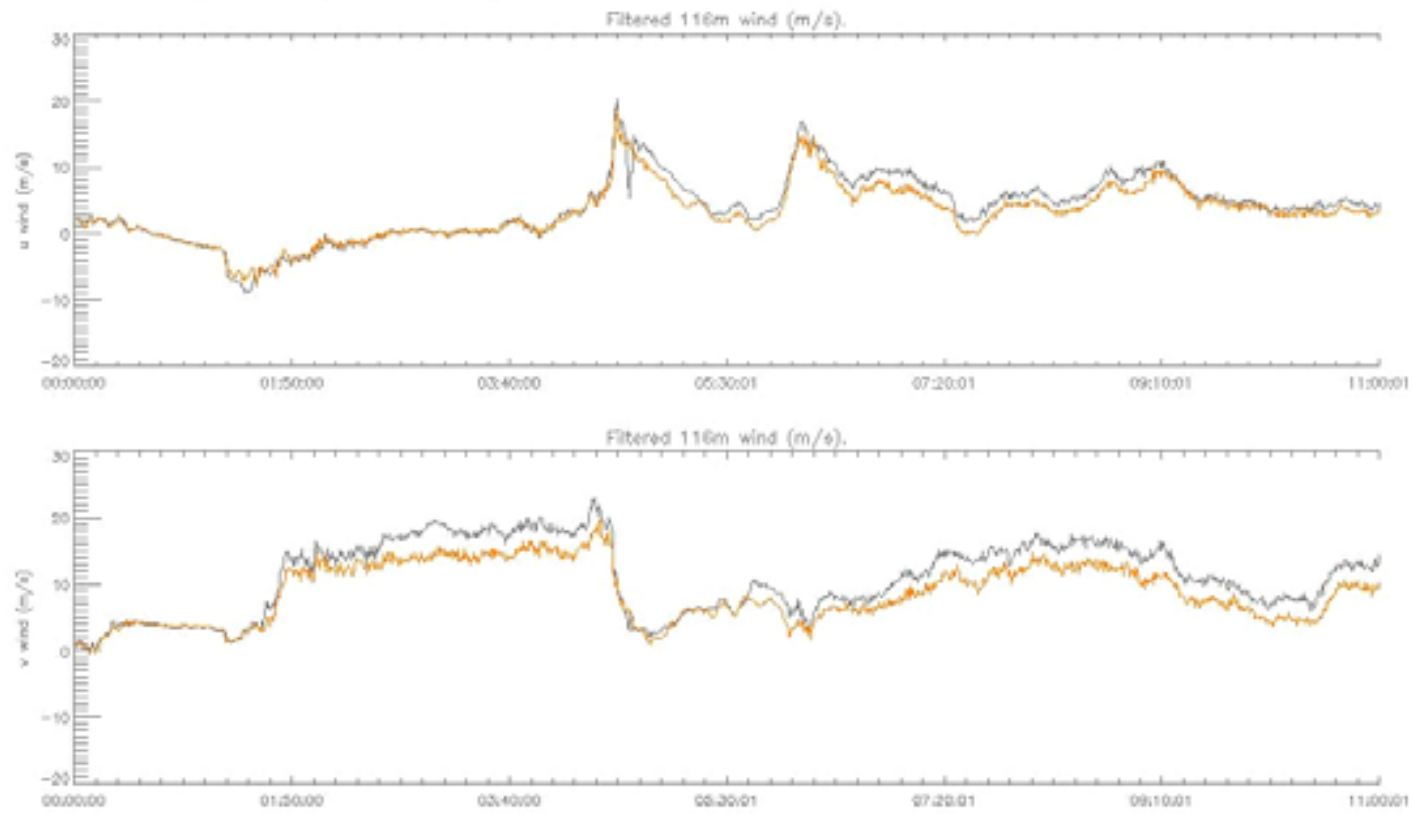

Filtered $116 \mathrm{~m}$ wind $(\mathrm{m} / \mathrm{s})$.

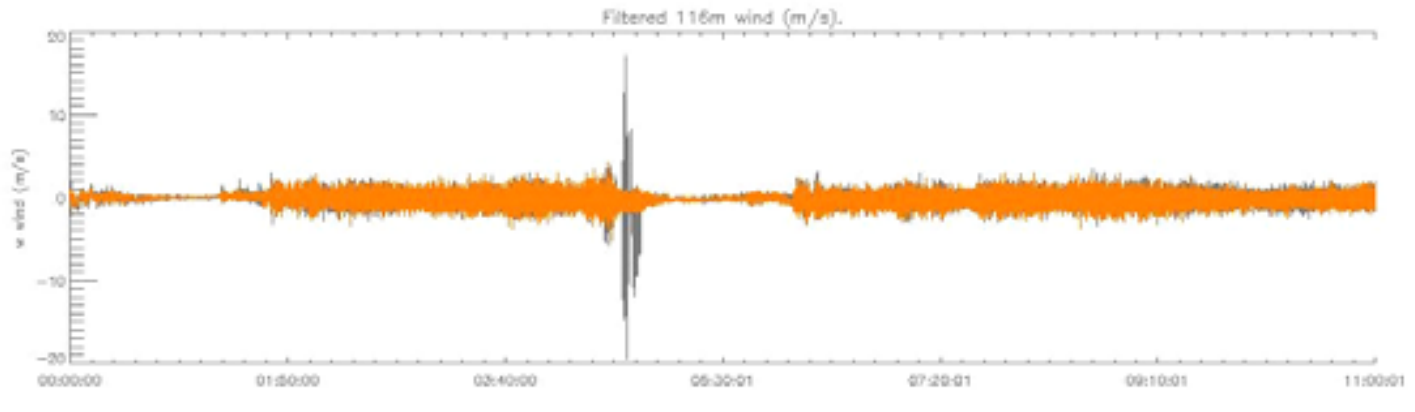

Figure 5. Horizontal winds at 54 (orange) and $116 \mathrm{~m}$ (gray) averaged for $30 \mathrm{~s}$ from the data obtained on September 10, 2003 ( $u$ top, $v$ middle). Comparison with the vertical velocities at $10 \mathrm{~Hz}$ (lower panel and Figure 4a) shows a clear correlation of turbulence with significant mean wind shear and small $R i$. Turbulence is largely absent even when significant shears are present when $R i>1 / 4$.

Stretched views of the data that span the fronts on September 13 and 14 are provided in Figure 6 . In each case, we see significant oscillatory behavior that is probably wave-like at longer periods and for $R i \sim 0$ or greater. There is the likelihood of the existence of instability structures occurring at smaller scales and periods that provide the mechanism to transfer kinetic energy to turbulence scales. Figures $6 \mathrm{a}$ and $6 \mathrm{~b}$ exhibit oscillatory variations in vertical velocity that have observed periods of 3-5 min with upward motions that correlate with maxima in the component vorticities and CTKE. Also, smaller scale oscillations (primarily Figures 6a and 6c) indicate instability structures that are both the triggers and the energy sources for turbulence at smaller scales. In each case, the frontal response appears to be nearly coherent across the depth of the boundary layer. 
Lamar Tower: 13 Sep 2003: Ri Layer $=67-85 \mathrm{~m}$. Vorticity layer $=67 \mathrm{~m}$

Ri Filtering $=5 \mathrm{~s}$ ave every $5 \mathrm{~s}$. Vorticity filtering $=0.10 \mathrm{~s}$ ave every $0.10 \mathrm{~s}$.

Average velocity $=16.30 \mathrm{~m} / \mathrm{s}$
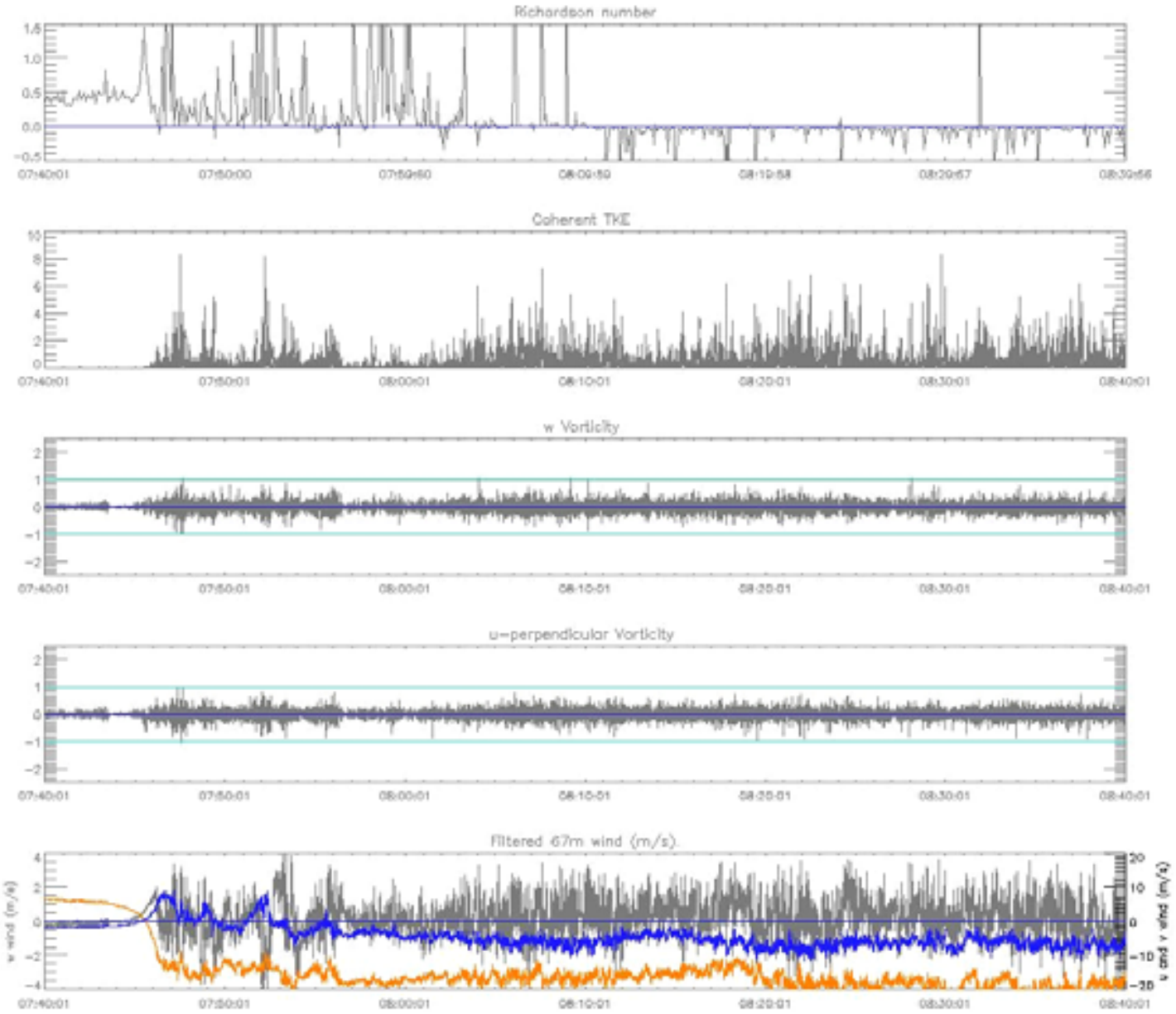

Figure 6a. As in Figure 4b, but expanding the data from 0740 to 0840 UT. 
Lamar Tower: 14 Sep 2003: Ri Layer $=67-85 \mathrm{~m}$. Vorticity layer $=67 \mathrm{~m}$

Ri Filtering $=5 \mathrm{~s}$ ave every $5 \mathrm{~s}$. Vorticity filtering $=0.10 \mathrm{~s}$ ave every $0.10 \mathrm{~s}$.

Average velocity $=11.70 \mathrm{~m} / \mathrm{s}$
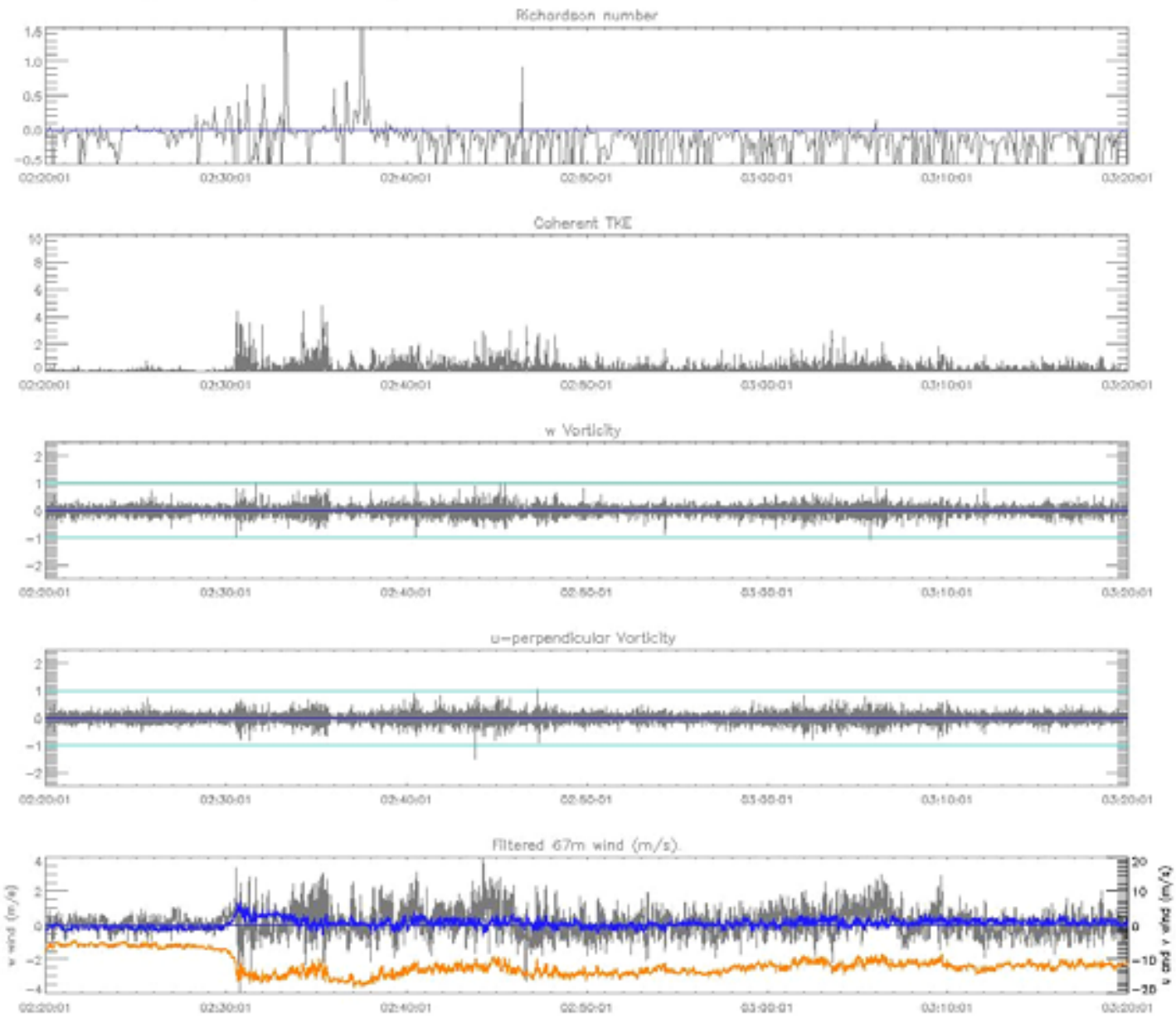

Figure 6b. As in Figure 4c, but expanding the data from 0220 to 0320 UT. 
Lamar Tower: 14 Sep 2003: Ri Layer $=67-85 \mathrm{~m}$. Vorticity layer $=67 \mathrm{~m}$

Ri Filtering $=5 \mathrm{~s}$ ave every $5 \mathrm{~s}$. Vorticity filtering $=0.10 \mathrm{~s}$ ave every $0.10 \mathrm{~s}$.

Average velocity $=6.65 \mathrm{~m} / \mathrm{s}$

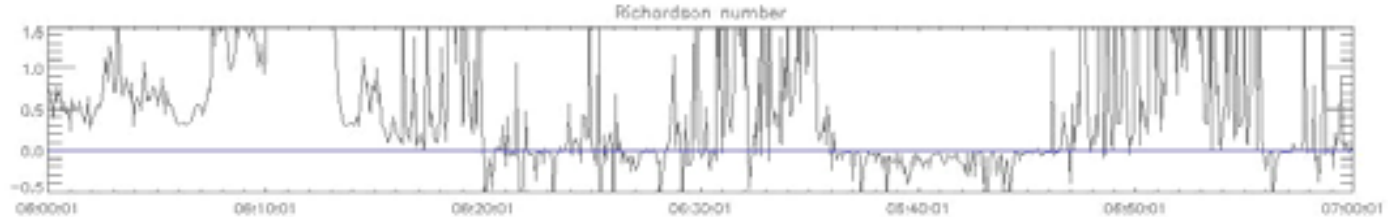

Coherent TKE
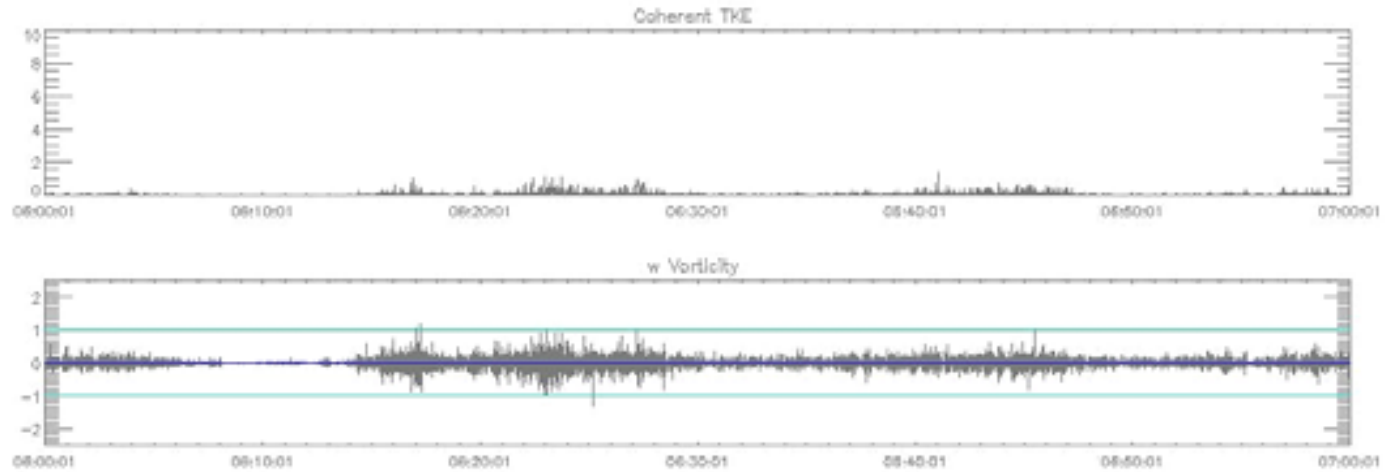

u-perpendicular Yorticity

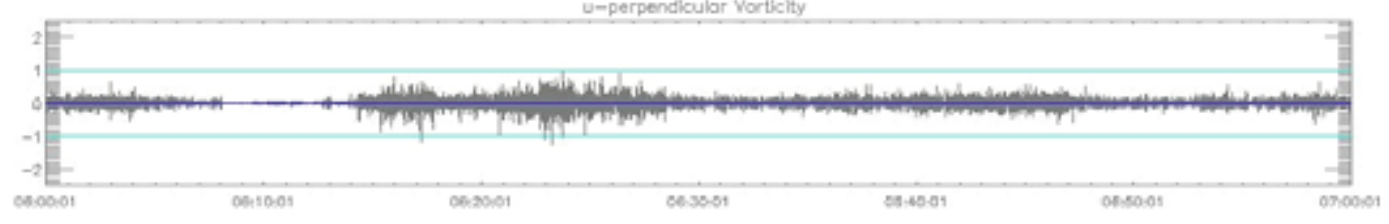

Fitered $67 \mathrm{~m}$ wind $\langle\mathrm{m} / \mathrm{s})$

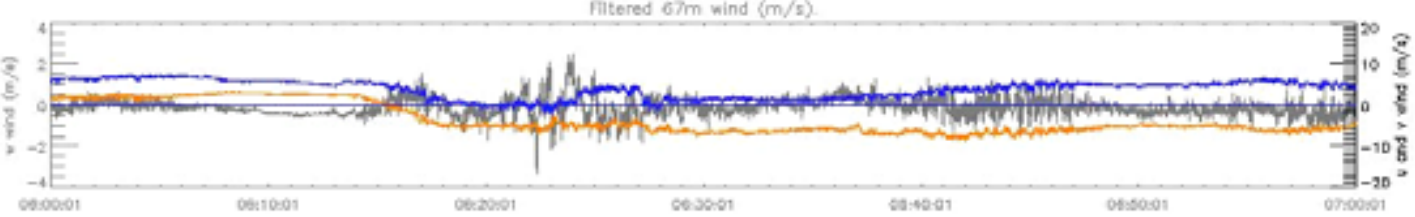

Figure 6c. As in Figure 4c, but expanding the data from 0600 to 0700 UT.

The small-scale oscillations observed following the frontal passage on September 13 are shown on an expanded scale in Figure 7, which shows that each of the three velocity components at altitudes of 85 and $116 \mathrm{~m}$ for 10 min spans the frontal passage. These reveal a sustained vertical shear of the zonal (E-W) wind of 2-3 m/s extending until the time at which significant oscillations begin. These oscillations exhibit temporal and horizontal spatial scales (based on advection) of 3-30 s and 50-500 m, respectively. Indeed, of the 2003 Lamar data analyzed, these motions are the strongest, apart from the very large and localized vertical velocities that occurred at $\sim 0430$ UT on September 10 (see Figure 5, bottom). 


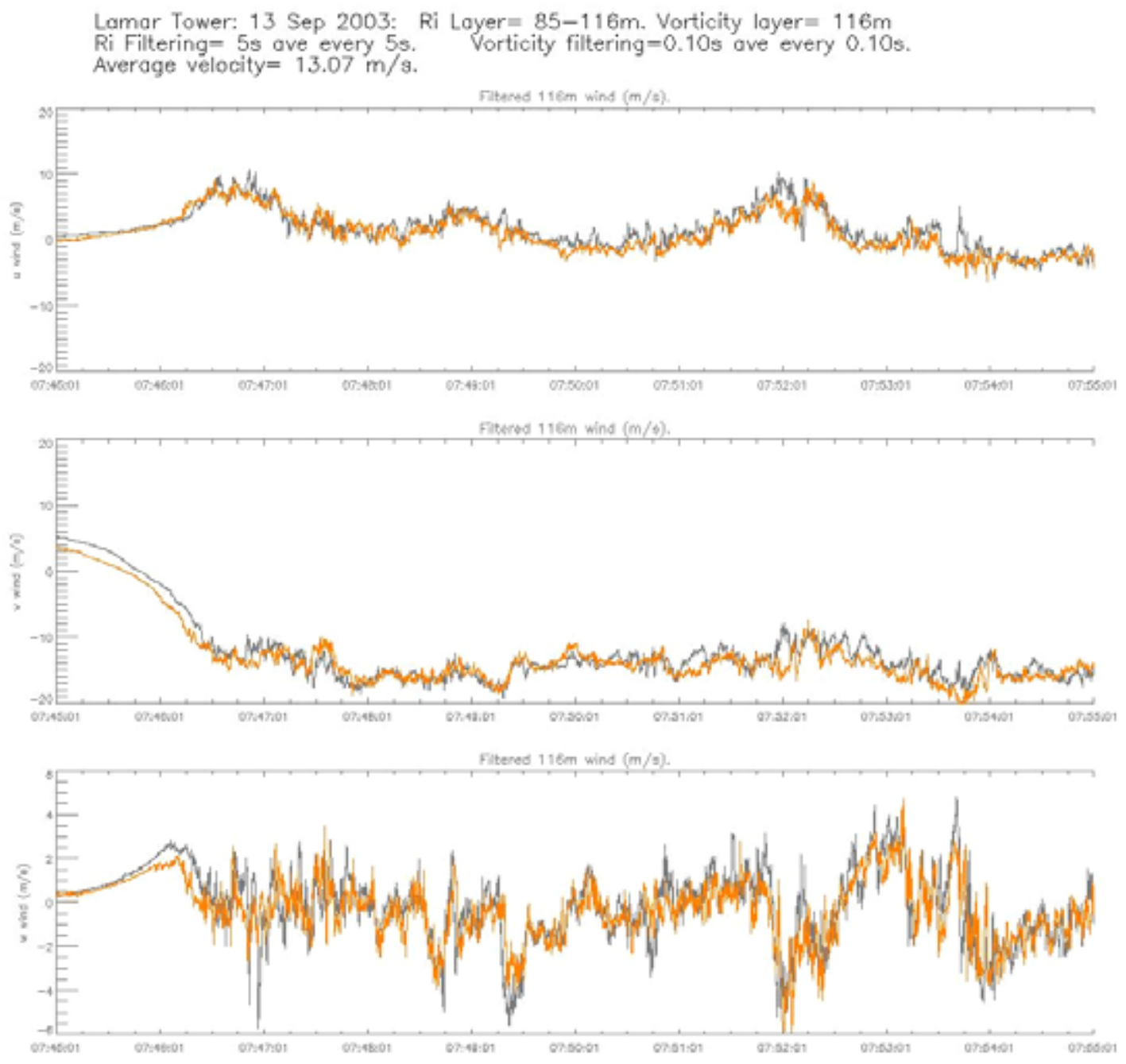

Figure 7. Component velocities ( $u$ top, $v$ middle, $w$ bottom) for September 13, 2003 from 0745 to 0755 UT at each of the four altitudes (orange at $85 \mathrm{~m}$, gray at $116 \mathrm{~m}$ ) show the evolution of the mean and oscillating motions that accompany frontal passage. Note the meridional wind shear between the two levels as the front passes the site.

\section{Focused Interval from Anemometer Array}

This short (11-min) interval of data collected at the NWTC on February 5, 2000 was acquired from a planar array of five sonic anemometers immediately upwind of an operating $600 \mathrm{~kW}$ wind turbine with a 43-m rotor diameter [Kelley et al. 2002]. It was selected and analyzed because it resulted in strong adverse wind turbine loading for which correlative boundary layer measurements were available. These data, shown in Figure 8 at 15, 37, and $58 \mathrm{~m}$, correspond to the turbine rotor lowest, hub, and highest elevations. The most striking features in these data are the very large oscillations in the velocities, particularly the vertical velocity, and the corresponding large values of component vorticities, especially $C T K E$. Large vertical velocities also occurred in Lamar on September 13, 2003 (see Figures 4b and 5a), but did not exceed $\sim 4 \mathrm{~m} / \mathrm{s}$. Extremely 
large vertical velocities were also seen in Lamar on September 10, but only for a limited duration. In this data set, vertical velocities as large as $\sim 7 \mathrm{~m} / \mathrm{s}$ are seen, primarily at the highest altitude. These component velocities are shown separately in Figure 9 in an expanded scale to exhibit both the amplitudes and dominant periods.

NWTC Array: 5 February 2000: Ri Layer $=15-37 \mathrm{~m}$. Vorticity layer $=15 \mathrm{~m} \square$

Ri Filtering $=5 \mathrm{~s}$ ave every $5 \mathrm{~s}$. Vorticity filtering $=0.1 \mathrm{~s}$ ave every $0.10 \mathrm{~s}$.

Average velocity= $11.22 \mathrm{~m} / \mathrm{s}$
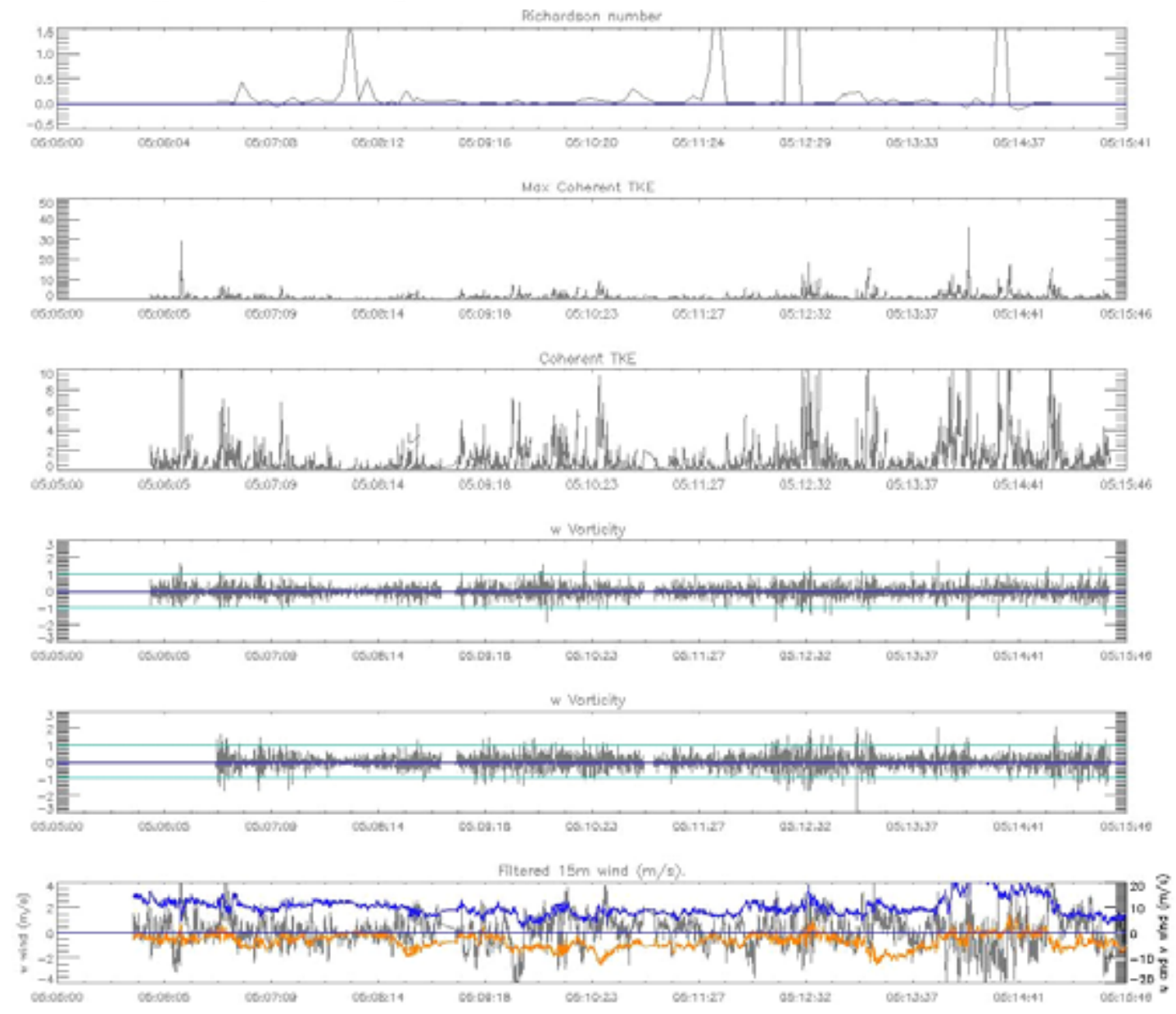

Figure 8a. As in Figure 6, but for NWTC data from 0505 to 0516 UT on February 5, 2000 at $15 \mathrm{~m}$. CTKE is plotted on two scales (maximum values of 50 and $10 \mathrm{~m}^{2} / \mathrm{s}^{2}$, second and third panels from top) to display both larger and smaller values. 
NWTC Array: 5 February 2000: Ri Layer $=37-61 \mathrm{~m}$. Vorticity layer $=37 \mathrm{~m}$

Ri Filtering $=5 \mathrm{~s}$ ave every $5 \mathrm{~s}$. Vorticity filtering $=0.1 \mathrm{~s}$ ave every $0.10 \mathrm{~s}$.

Average velocity $=12.17 \mathrm{~m} / \mathrm{s}$
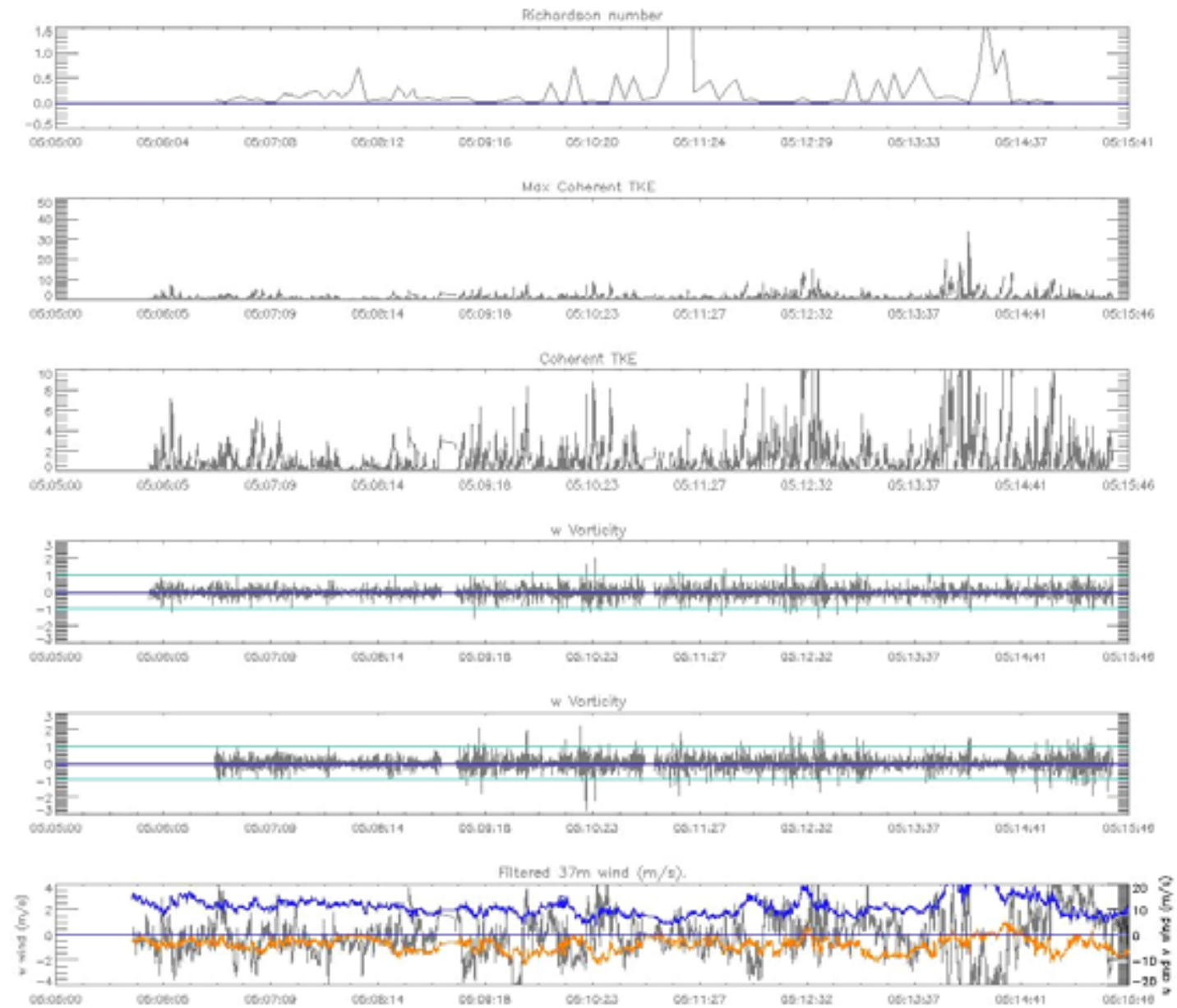

Figure 8b. As in Figure 8a, but for data at $37 \mathrm{~m}$. 
NWTC Array: 5 February 2000: Ri Layer $=37-61 \mathrm{~m}$. Vorticity layer $=61 \mathrm{~m}$

Ri Filtering $=5 \mathrm{~s}$ ave every $5 \mathrm{~s}$. Vorticity filtering $=0.1 \mathrm{~s}$ ave every $0.10 \mathrm{~s}$.

Average velocity $=12.17 \mathrm{~m} / \mathrm{s}$
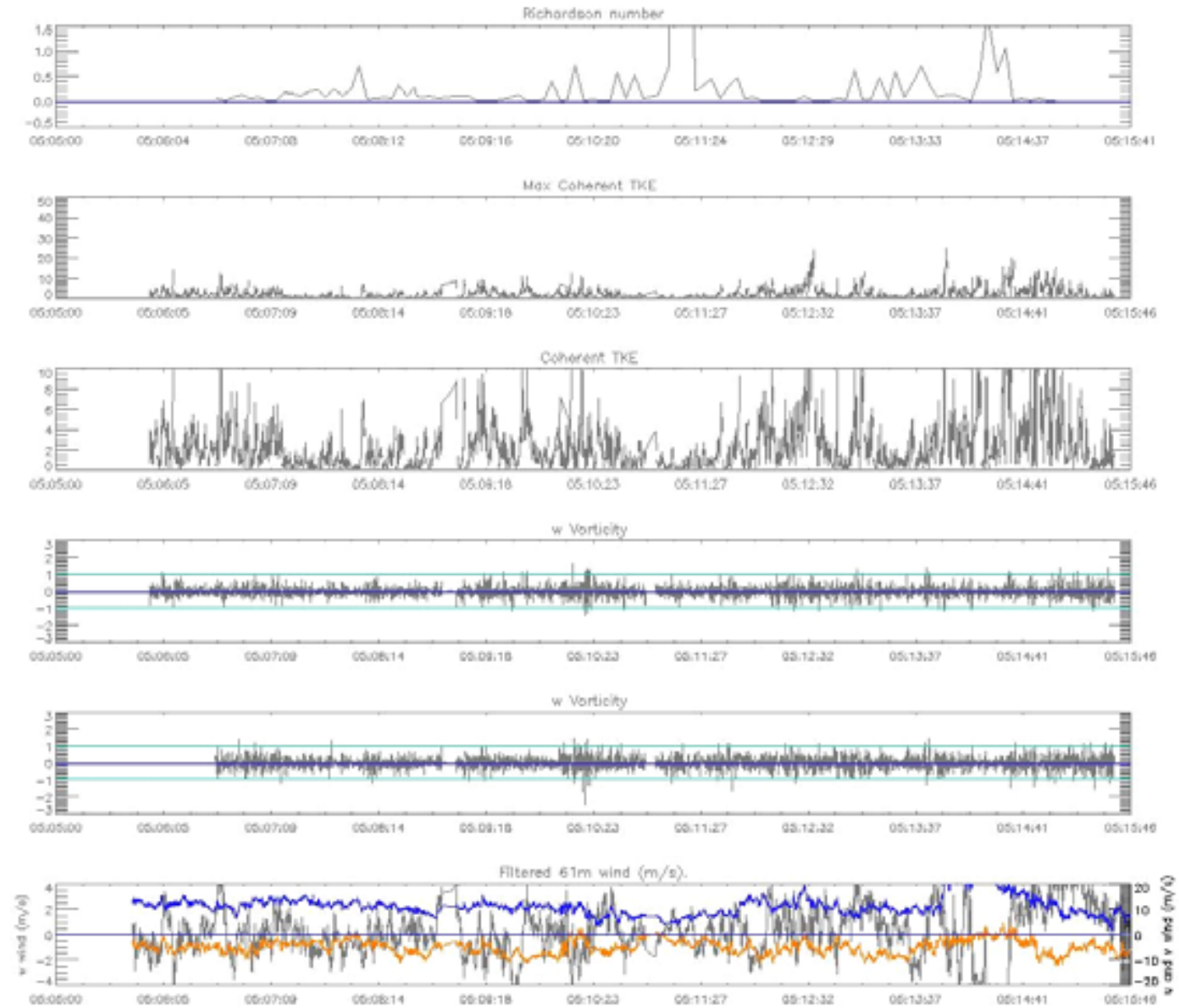

Figure 8c. As in Figure 8a, but for data at $61 \mathrm{~m}$. 
NWTC Array: 5 February 2000: Ri Layer $=37-61 \mathrm{~m}$. Vorticity layer $=37 \mathrm{~m}$

Ri Filtering $=5 \mathrm{~s}$ ave every $5 \mathrm{~s}$. Vorticity filtering $=0.1 \mathrm{~s}$ ave every $0.10 \mathrm{~s}$.

Average velocity $=15.24 \mathrm{~m} / \mathrm{s}$
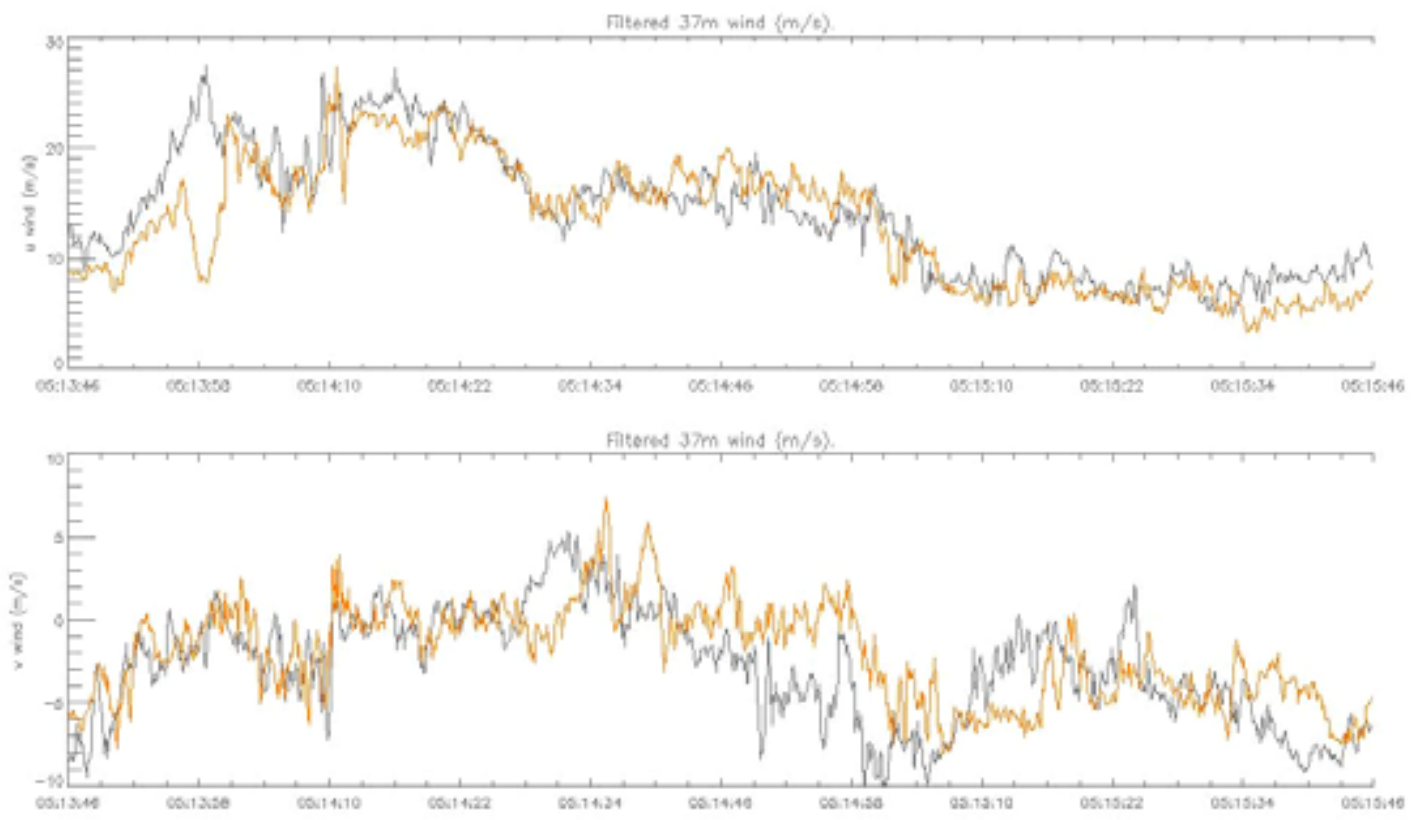

Filtered $37 \mathrm{~m}$ wind $\{\mathrm{m} / \mathrm{s}\rangle$

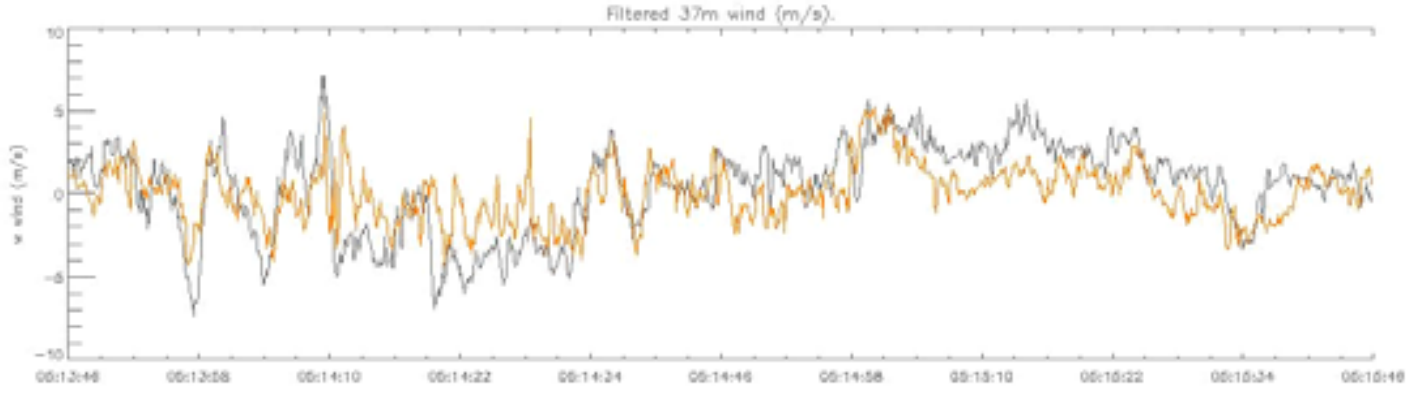

Figure 9. As in Figure 7 for February 5, 2000 from 0513:46 to 0515:46 UT at the two upper altitudes (orange at $37 \mathrm{~m}$, gray at $61 \mathrm{~m}$ ) show the evolution of the mean and oscillating motions.

The intervals with the largest velocities, vorticities, and CTKE typically also have $R i$ near zero or slightly positive. This strongly suggests that these are KH shear instabilities that have evolved because of the large mean shear and small mean stability that precede these oscillations. The small periods and spatial scales of the dominant oscillations, typically 1-6 s and 20-120 $\mathrm{m}$ (the larger scales and greater coherence occur at the earlier times), the large CTKE (and coherent horizontal and vertical velocities), and the development of a very large zonal wind shear $(10 \mathrm{~m} / \mathrm{s}$ or greater $37-58 \mathrm{~m})$ before and accompanying the onset of the large oscillations, confirm this finding.

\section{Results of Statistical Analysis}

Results of the CASES-99 and Lamar statistical analyses are displayed in Tables 1-3. Vertical resolution was the highest (below $85 \mathrm{~m}$ ) at the lower altitudes, so we expect to have the best ability here to characterize the stability $(R i)$ of the mean state. Most of the 
large cross-stream vorticity (thus also velocity fluctuations and CTKE) occurred for mean $R i<0(157 / 217$ levels and days, or $72 \%)$. An even larger fraction (193/217 levels and days, or $89 \%$ ) had $R i<0.05$.

Table 1. Number of days when the maximum number of points with vorticity magnitude greater than $1(\mathrm{~m} / \mathrm{s} / \mathrm{m})$ was within each $R i$ range. A day consists of all data between 00 and 11 UTC. The "Days" column shows the total number of days available at each level for the Lamar (L) and Cases-99 (C) tower data.

\begin{tabular}{|c|c|c|c|c|c|c|c|}
\hline Level & Days & $R i<0$ & $\begin{array}{l}R i= \\
0-.05\end{array}$ & $\begin{array}{l}R i= \\
.05-.10\end{array}$ & $\begin{array}{l}R i= \\
.10-.25\end{array}$ & $\begin{array}{l}R i= \\
0.25-1\end{array}$ & $R i>1$ \\
\hline $116 \mathrm{~m}$ & L 15 & 2 & 0 & 1 & 2 & 8 & 2 \\
\hline $85 \mathrm{~m}$ & L 13 & 0 & 0 & 3 & 6 & 1 & 3 \\
\hline $67 \mathrm{~m}$ & L 15 & 2 & 8 & 1 & 3 & 1 & 0 \\
\hline $55 \mathrm{~m}$ & C 22 & 21 & 1 & 0 & 0 & 0 & 0 \\
\hline $54 \mathrm{~m}$ & L 15 & 5 & 5 & 0 & 3 & 2 & 0 \\
\hline $50 \mathrm{~m}$ & C 22 & 20 & 2 & 0 & 0 & 0 & 0 \\
\hline $40 \mathrm{~m}$ & C 23 & 18 & 3 & 1 & 0 & 1 & 0 \\
\hline $30 \mathrm{~m}$ & C 24 & 4 & 12 & 0 & 1 & 7 & 0 \\
\hline $20 \mathrm{~m}$ & C 24 & 21 & 2 & 0 & 0 & 1 & 0 \\
\hline $10 \mathrm{~m}$ & C 24 & 22 & 1 & 0 & 1 & 0 & 0 \\
\hline $5 \mathrm{~m}$ & C 24 & 22 & 1 & 0 & 1 & 0 & 0 \\
\hline $1.5 \mathrm{~m}$ & C 24 & 22 & 1 & 0 & 1 & 0 & 0 \\
\hline
\end{tabular}


Table 2. Total component vorticity means (upper left value), standard deviations (upper right value), and number of points (lower value) contributing for each CTKE range (top of each column) and altitude. Units are $\mathrm{m} / \mathrm{s}$ and $\mathrm{m}^{2} / \mathrm{s}^{2}$ for component vorticity and CTKE, respectively. See text for discussion.

\begin{tabular}{|c|c|c|c|c|}
\hline Level & CTKE 1 to 2 & CTKE 2 to 5 & CTKE 5 to 10 & $C T K E \geq 10$ \\
\hline $116 \mathrm{~m}$ & $\begin{array}{l}0.284(0.488) \\
39821\end{array}$ & $\begin{array}{l}0.685(1.078) \\
10987\end{array}$ & $\begin{array}{l}1.655(1.623) \\
1868\end{array}$ & $\begin{array}{l}2.264(1.915) \\
810\end{array}$ \\
\hline $85 \mathrm{~m}$ & $\begin{array}{l}0.212(0.163) \\
47372\end{array}$ & $\begin{array}{l}0.225(0.171) \\
9313\end{array}$ & $\begin{array}{l}0.295(0.219) \\
265\end{array}$ & $\begin{array}{l}0.284(0.076) \\
5\end{array}$ \\
\hline $67 \mathrm{~m}$ & $\begin{array}{l}0.231(0.178) \\
52760\end{array}$ & $\begin{array}{l}0.242(0.183) \\
9413\end{array}$ & $\begin{array}{l}0.256(0.194) \\
295\end{array}$ & $\begin{array}{l}0.981(\quad) \\
1\end{array}$ \\
\hline $55 \mathrm{~m}$ & $\begin{array}{l}0.268(0.207) \\
247186\end{array}$ & $\begin{array}{l}0.287(0.219) \\
60959\end{array}$ & $\begin{array}{l}0.302(0.225) \\
3194\end{array}$ & $\begin{array}{l}0.338(0.271) \\
165\end{array}$ \\
\hline $54 \mathrm{~m}$ & $\begin{array}{l}0.232(0.172) \\
54657\end{array}$ & $\begin{array}{l}0.248(0.183) \\
9531\end{array}$ & $\begin{array}{l}0.286(0.195) \\
261\end{array}$ & $\begin{array}{l}0.366(0.077) \\
4\end{array}$ \\
\hline $50 \mathrm{~m}$ & $\begin{array}{l}0.300(0.240) \\
229023\end{array}$ & $\begin{array}{l}0.320(0.248) \\
55321\end{array}$ & $\begin{array}{l}0.361(0.321) \\
2608\end{array}$ & $\begin{array}{l}0.488(0.459) \\
119\end{array}$ \\
\hline $40 \mathrm{~m}$ & $\begin{array}{l}0.292(0.223) \\
257098\end{array}$ & $\begin{array}{l}0.310(0.232) \\
71231\end{array}$ & $\begin{array}{l}0.343(0.265) \\
4317\end{array}$ & $\begin{array}{l}0.363(0.267) \\
169\end{array}$ \\
\hline $30 \mathrm{~m}$ & $\begin{array}{l}0.343(0.249) \\
283899\end{array}$ & $\begin{array}{l}0.372(0.265) \\
75189\end{array}$ & $\begin{array}{l}0.420(0.311) \\
4190\end{array}$ & $\begin{array}{l}0.579(0.533) \\
168\end{array}$ \\
\hline $20 \mathrm{~m}$ & $\begin{array}{l}0.321(0.246) \\
385025\end{array}$ & $\begin{array}{l}0.337(0.256) \\
128508\end{array}$ & $\begin{array}{l}0.383(0.561) \\
8942\end{array}$ & $\begin{array}{l}0.602(2.098) \\
360\end{array}$ \\
\hline $10 \mathrm{~m}$ & $\begin{array}{l}0.438(0.318) \\
360217\end{array}$ & $\begin{array}{l}0.470(0.346) \\
100132\end{array}$ & $\begin{array}{l}0.511(0.357) \\
5470\end{array}$ & $\begin{array}{l}0.565(0.361) \\
236\end{array}$ \\
\hline $5 \mathrm{~m}$ & $\begin{array}{l}0.568(0.402) \\
408971\end{array}$ & $\begin{array}{l}0.602(0.398) \\
121293\end{array}$ & $\begin{array}{l}0.656(0.426) \\
7148\end{array}$ & $\begin{array}{l}0.764(0.851) \\
275\end{array}$ \\
\hline $1.5 \mathrm{~m}$ & $\begin{array}{l}0.890(0.595) \\
386150\end{array}$ & $\begin{array}{l}0.939(0.636) \\
104130\end{array}$ & $\begin{array}{l}1.072(0.751) \\
5102\end{array}$ & $\begin{array}{l}1.352(1.400) \\
200\end{array}$ \\
\hline
\end{tabular}


Table 3. As in Table 2, but for vertical velocity magnitude.

\begin{tabular}{|c|c|c|c|c|}
\hline Level & CTKE 1 to 2 & CTKE 2 to 5 & CTKE 5 to 10 & CTKE $\geq 10$ \\
\hline $116 \mathrm{~m}$ & $\begin{array}{l}1.012(0.881) \\
39821\end{array}$ & $\begin{array}{l}1.641(1.580) \\
10987\end{array}$ & $\begin{array}{l}3.078(2.775) \\
1868\end{array}$ & $\begin{array}{l}4.604(3.522) \\
810\end{array}$ \\
\hline $85 \mathrm{~m}$ & $\begin{array}{l}0.914(0.610) \\
47372\end{array}$ & $\begin{array}{l}1.220(0.752) \\
9313\end{array}$ & $\begin{array}{l}1.717(0.851) \\
265\end{array}$ & $\begin{array}{l}2.502(0.662) \\
5\end{array}$ \\
\hline $67 \mathrm{~m}$ & $\begin{array}{l}0.923(0.633) \\
52760\end{array}$ & $\begin{array}{l}1.232(0.782) \\
9413\end{array}$ & $\begin{array}{l}1.481(0.860) \\
295\end{array}$ & $\begin{array}{l}5.042(\quad) \\
1\end{array}$ \\
\hline $55 \mathrm{~m}$ & $\begin{array}{l}1.016(0.692) \\
247186\end{array}$ & $\begin{array}{l}1.315(0.847) \\
60959\end{array}$ & $\begin{array}{l}1.805(1.092) \\
3194\end{array}$ & $\begin{array}{l}1.777(1.320) \\
165\end{array}$ \\
\hline $54 \mathrm{~m}$ & $\begin{array}{l}0.904(0.567) \\
54657\end{array}$ & $\begin{array}{l}1.238(0.727) \\
9531\end{array}$ & $\begin{array}{l}1.648(0.898) \\
261\end{array}$ & $\begin{array}{l}2.400(0.467) \\
4\end{array}$ \\
\hline $50 \mathrm{~m}$ & $\begin{array}{l}0.957(0.683) \\
229023\end{array}$ & $\begin{array}{l}1.245(0.852) \\
55321\end{array}$ & $\begin{array}{l}1.744(1.124) \\
2608\end{array}$ & $\begin{array}{l}1.948(1.313) \\
119\end{array}$ \\
\hline $40 \mathrm{~m}$ & $\begin{array}{l}0.989(0.670) \\
257098\end{array}$ & $\begin{array}{l}1.253(0.819) \\
71231\end{array}$ & $\begin{array}{l}1.698(1.074) \\
4317\end{array}$ & $\begin{array}{l}2.075(1.181) \\
169\end{array}$ \\
\hline $30 \mathrm{~m}$ & $\begin{array}{l}0.919(0.638) \\
283899\end{array}$ & $\begin{array}{l}1.204(0.806) \\
75189\end{array}$ & $\begin{array}{l}1.691(1.057) \\
4190\end{array}$ & $\begin{array}{l}2.105(1.266) \\
168\end{array}$ \\
\hline $20 \mathrm{~m}$ & $\begin{array}{l}0.867(0.598) \\
385025\end{array}$ & $\begin{array}{l}1.069(0.732) \\
128508\end{array}$ & $\begin{array}{l}1.405(0.947) \\
8942\end{array}$ & $\begin{array}{l}1.608(1.107) \\
360\end{array}$ \\
\hline $10 \mathrm{~m}$ & $\begin{array}{l}0.872(0.567) \\
360217\end{array}$ & $\begin{array}{l}1.118(0.702) \\
100132\end{array}$ & $\begin{array}{l}1.488(0.893) \\
5470\end{array}$ & $\begin{array}{l}2.178(1.310) \\
236\end{array}$ \\
\hline $5 \mathrm{~m}$ & $\begin{array}{l}0.762(0.510) \\
408971\end{array}$ & $\begin{array}{l}0.989(0.650) \\
121293\end{array}$ & $\begin{array}{l}1.319(0.860) \\
7148\end{array}$ & $\begin{array}{l}1.532(1.878) \\
275\end{array}$ \\
\hline $1.5 \mathrm{~m}$ & $\begin{array}{l}0.651(0.430) \\
386150\end{array}$ & $\begin{array}{l}0.833(0.547) \\
104130\end{array}$ & $\begin{array}{l}1.033(0.717) \\
5102\end{array}$ & $\begin{array}{l}1.166(1.324) \\
200\end{array}$ \\
\hline
\end{tabular}


Only $\sim 11 \%$ of significant turbulence occurred for $R i>0.05$, despite the potential for shear instability at higher values. This is almost certainly an overestimation, given that we could not define $R i$ well with coarsely spaced instrumentation. Only at the highest tower altitudes (and only at Lamar) did we observe departures from these statistics. Most high vorticity occurred at 85 and $116 \mathrm{~m}$ altitudes for $R i>0.10$ and 0.25 , respectively. But these altitudes were also those where $R i$ was estimated over a $31-\mathrm{m}$ interval rather than over intervals of 3.5-10 m, with one interval of $18 \mathrm{~m}$, at lower levels.

Tables 2 and 3 show the mean values of total component vorticity and vertical velocity magnitude for various ranges of CTKE. Most CTKE estimates were less than $1 \mathrm{~m}^{2} / \mathrm{s}^{2}$, even in significant turbulence as seen in the component vorticity and velocity data. Of those values greater than $1 \mathrm{~m}^{2} / \mathrm{s}^{2}$, nearly $80 \%$ were less than $2 \mathrm{~m}^{2} / \mathrm{s}^{2}$, with only $1 \%$ greater than $5 \mathrm{~m}^{2} / \mathrm{s}^{2}$ and $0.04 \%$ greater than $10 \mathrm{~m}^{2} / \mathrm{s}^{2}$. Peak values in Lamar on September 10,13 , and 14 were as high as $5-10 \mathrm{~m}^{2} / \mathrm{s}^{2}$, and several maxima that occurred at the NWTC on February 5, 2001 were as high as $\sim 30 \mathrm{~m}^{2} / \mathrm{s}^{2}$.

The results displayed in Tables 2 and 3 indicate a correlation of turbulence intensity, as measured by CTKE, relative to measures based on component vorticities and vertical velocities. At $85 \mathrm{~m}$ and below, CTKE increases from the lowest to the highest range (an increase of $\sim 10$ ) correspond to component vorticity and vertical velocity increases of $<2$ to $\sim 3$, with a mean increase of $\sim 2$. This is equivalent to enstrophy (vorticity variance) and vertical velocity variance increases of $\sim 4$. At $116 \mathrm{~m}$, the fractional increases of component vorticity and vertical velocity are $\sim 10$ and 4 , respectively, which suggests that at these altitudes, CTKE is less sensitive, relative to enstrophy or vertical velocity variance, than at lower altitudes. Thus CTKE is statistically more sensitive to strong turbulence at lower altitudes, but less so at the highest altitudes. This suggests that no single measure of turbulence intensity is clearly superior to the others. In particular, CTKE may not be an unbiased measure of turbulence, as it measures turbulent (and larger scale) fluxes of momentum, rather than turbulence intensity at specific scales. Because CTKE is correlated with the other measures we have evaluated (including TKE, see Figure 14); however, all are likely suitable to characterize turbulence intensity over a suitably averaged volume.

The full story may be somewhat more complicated, because coherent structures at larger spatial scales may play important roles in adverse loading of wind turbine blades. Indeed, there are good reasons to suspect that large vertical motions, which appear to contribute significantly to large CTKE, may be strong diagnostics because of their direct implications for varying angle of attack relative to the incident flow and for dynamic loading resulting from nonzero Reynolds stresses (and CTKE) at larger scales. 


\section{DNS KHI Data Analysis Methodology}

\section{DNS KHI Evolution}

A high-resolution Direct Numerical Simulation (DNS) of KHI, at varying $R e$ and $R i$, has been completed as part of Air Force-, U.S. Department of Energy-, and National Science Foundation-sponsored atmospheric turbulence studies. In order to examine the role of stratification, which gives rise to intense, vertically isolated shear layers in the atmosphere, we conducted the DNS work with no turbulence modeling. Such subgridscale (SGS) modeling is problematic under stable conditions; as a result, certain important morphological features are inadequately described with turbulence modeling. An example is shown in the top two rows of Figure 10, which clearly depict the emergence and subsequent breakdown of coherent streamwise-aligned vortex structures that result from a secondary (convective) instability near the edges of the primary KHI billow (or rotor). At later stages of the KHI evolution, vortex structures are less organized, but there is a tendency for enhanced spanwise vorticity (aligned in the plane of turbine blade motions) both within the billow core (the more intense regions of energy dissipation in the bottom middle and right images) and in the regions of high thermal dissipation (with high thermal and wind gradients) at the edges of the turbulent layer.
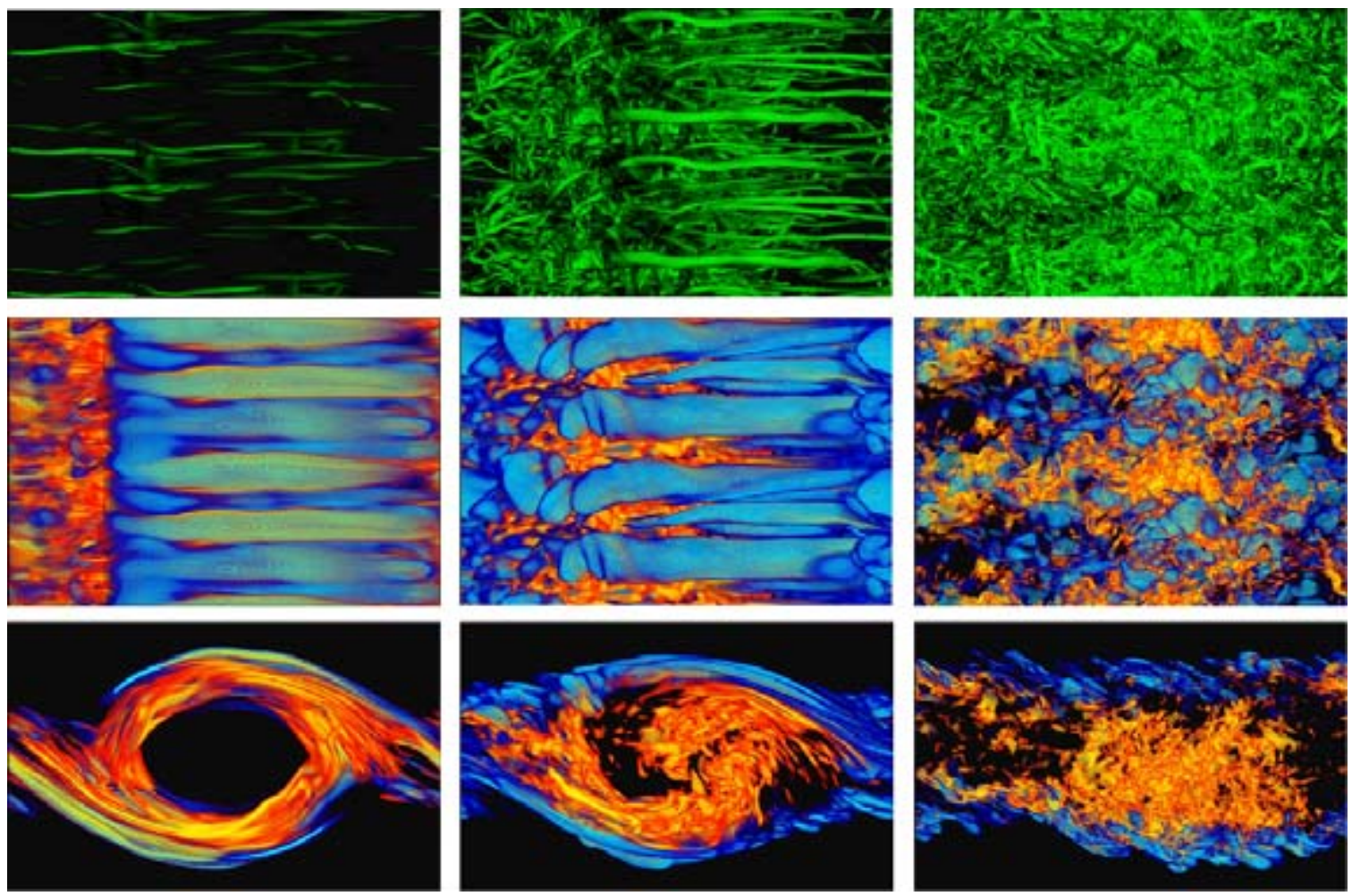

Figure 10. Volumetric views of instability and turbulence caused by $\mathrm{KHI}$ show vortex structures (top, viewed from above), mechanical (yellow-orange), and thermal (blue) dissipation viewed from above (middle) and from the side (bottom). The images are during secondary instability (left), early mixing of coherent billow core (center), and of the turbulent layer following billow breakdown (right). 
The scales of the initial streamwise vortex tubes are comparable to the initial shear depth when they emerge from the background flow, and they persist in a coherent form as they shrink to $\sim 15 \%$ of this initial width. These streamwise vortex-tube features are apparent throughout the shear layer, but they are most coherent and concentrate predominantly near the edges of the layer where the flow is best described as a transitional zone between turbulent and laminar motion. Because SGS modeling cannot yet adequately describe strongly stratified transitional flows, we expect the secondary instability (and any associated dynamic turbine loading) captured in detail here will be largely missing for most moderate-resolution liquid encapsulated synthesis Large-Eddy Simulation (LES) models.

When scaling the simulation depicted in Figure 10 for a 70-m deep billow and assuming a layer $R e$ of $R e=10^{7}\left(10^{8}\right)$, the velocity difference across the layer is $2 U_{o}=2 \mathrm{~m} / \mathrm{s}(20$ $\mathrm{m} / \mathrm{s}$ ), and the characteristic unit of time is $h / U_{o}=12 \mathrm{~s}(1.2 \mathrm{~s})$. Here $h$ is a unit of length equal to $\sim 1 / 6$ of the full layer depth. With these units, the secondary instability develops in roughly $10 \mathrm{~min}(1 \mathrm{~min})$ of evolution and persists in a strong coherent form for another $20 \mathrm{~min}(2 \mathrm{~min})$. The intensity and scale of the coherent vortex structures decay for these "run-down" simulations, with a decay time of $70 \mathrm{~min}(7 \mathrm{~min})$ for an unforced wind shear event. Such responses would be more persistent if they were continuously shed by an upstream orographic forcing feature.

\section{DNS KHI Data Provision for Turbine Loading Model}

We provided velocity fields from a DNS of KHI to NREL to evaluate turbine loading in a realistic KHI environment. These data were configured somewhat differently from the LES results provided to NREL by Drs. Peter Sullivan and Ned Patton of the National Center for Atmospheric Research because of the very large data sets and limited temporal resolution of the DNS data. For example, the DNS that yielded the results shown in Figure 10 required as many as $1200 \times 400 \times 2400$ spectral modes and 150,000 CPU hours of Cray T3E time, and the results represent several terabytes of data. Because advective changes in vortex dynamics are typically much larger than local temporal changes $\left(U_{o}\right.$ $d u / d x>>d u / d t$, where derivatives are partial derivatives), we have provided 17 3-D velocity fields that span the range of important KHI turbulence flows to which a wind turbine will be subjected (including billow roll-up, secondary instability, turbulence growth and billow decay, turbulence decay, and restratification). They can also be employed to compute the correlation of strong turbine loading with coherent vortices and CTKE to assess the diagnostic potential of these quantities. These DNS results can be scaled for NREL's purposes up to $R e \sim 10^{7}$. For $R e \sim 10^{8}$ or larger, higher resolution simulations would need to be performed.

\section{Results of DNS KHI Analysis}

Our DNS of KHI revealed features of this source of atmospheric turbulence that may have implications for wind turbine fault, loading, and stress assessments. Among them are: 
- Vortex orientations and intensities that vary with position in the billow and its temporal evolution

- Mean streamwise shears (spanwise vorticity) that intensify strongly at the billow and subsequent turbulence layer edges

- Significant departures from the character and statistics of isotropic homogeneous turbulence, especially high intermittency, which imply more frequent intense vortices at smaller scales of motion.

Temperature and velocity variances throughout a KHI evolution (Figure 11) indicate that the two fields have structures that evolve very differently as a consequence of initial instability at the outer billow edges. The billow core remains a site of intense vortices with local "hotspots" of vorticity and mechanical energy dissipation to relatively late times. The billow edges have smaller mean vortex intensities, but form a major site of intermittent intense vortices at later stages as these edge regions continue to spawn tertiary instabilities at smaller spatial scales (see the lower middle and right panels of Figure 10). Streamwise vortex alignments are favored during initial instability in the billow edge regions. Spanwise vortex alignments accompany the reconnection regions of the initial streamwise vortices and are favored within the billow core (they retain significant large-scale spanwise vorticity) and in the edge regions at later stages after billow decay.
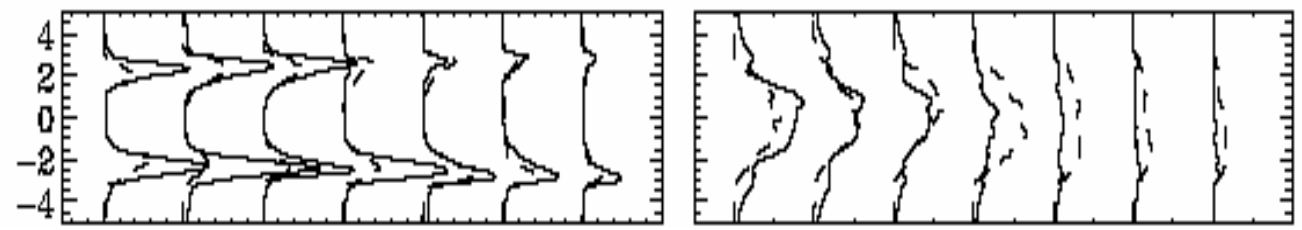

Figure 11. Temperature (left) and velocity (right) variance profiles for the DNS of KHI at times of $117,134,142,165,183,202$, and $220 h / U_{0}$. 2-D and 3-D contributions are shown with solid and dashed lines, respectively. Profiles are through the billow center.

The form of the turbulence spectrum can be obtained from either the spatial spectra or the second-order structure functions of $T, u, v$, and $w$. The averaged structure functions provide a more confident picture of spectral shape (see examples computed at $\mathrm{t}=183$ $h / U_{o}$ in Figure 12). For reference, a second-order structure function slope at large separations, $r$, of $\alpha=0.4$ corresponds to a power spectral slope of $-5 / 3$ that we expect for inertial-range turbulence. Departures from $\alpha=0.4$ imply steeper or shallower slopes and suggest stratification influences first hypothesized by Bolgiano (1959). In particular, shallower slopes, together with greater intermittency, imply that stronger and more frequent small-scale vortices may arise from larger scale KHI than implied by inertialrange turbulence statistics. Structure functions also reveal a lack of isotropy, with differing coefficients and inferred slopes for streamwise and spanwise separations, and differing variances of wind and wind shear components in the streamwise, spanwise, and vertical extending to the smallest scales within the inertial range. The results imply that stratification and shear influences extend even to these smallest scales. Stratification 
suppresses vertical velocities and enhances vertical shears, and mean shear lengthens turbulence scales in the streamwise compared to the spanwise directions.
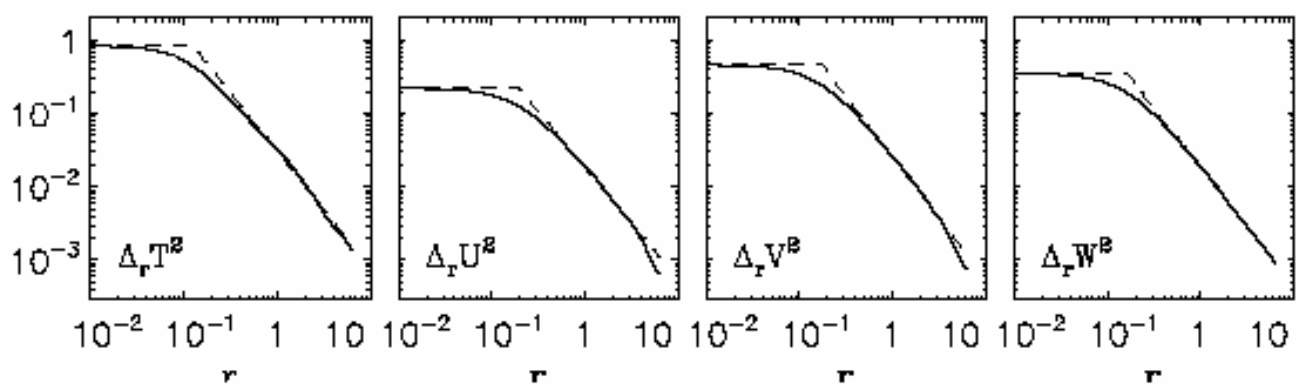

Figure 12. Compensated (i.e., divided by $r^{2}$ ) second-order structure functions for $T, u, v$, and $w$ near the middle of the shear layer at $t=183 \mathrm{~h} / \mathrm{U}_{0}$. Dashed lines show fits of the form $C_{A}{ }^{2} r^{\alpha}$ at separations above 3 times the inner scale. $A$ denotes the variable and $\alpha$ the slope which varies from $\sim 0.4$ to 0.66 for $T$ and $u$ and is somewhat smaller for $v$ and $w$.

The structure function parameters vary throughout the billow, both spatially and temporally. These results reveal steeper slopes, dramatically larger temperature structure functions, $C_{T}^{2}$, and inner scales in the edge regions of the billow and following turbulence layer (Figure 13).
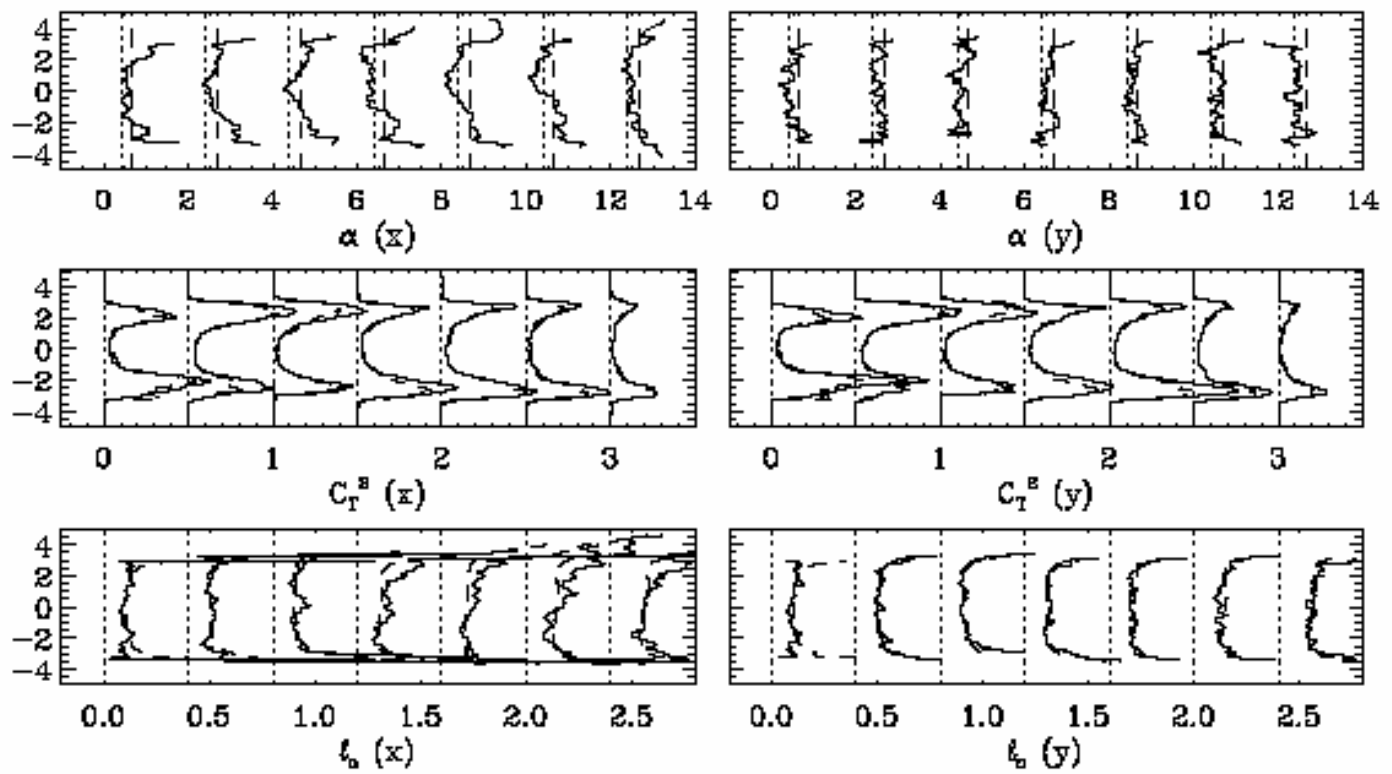

Figure 13. Profiles of structure-function fit parameters at $t=117,134,142,165,183$, 202 , and $220 h / U_{o}$. Left (right) panels show fits for streamwise (spanwise) separations. The top row shows $\alpha$ throughout the shear layer, with dotted (dashed) line showing $\alpha=$ 2/5 (2/3). Middle panels show $C_{T}{ }^{2}$ (solid lines) and $C_{T}{ }^{2}=C_{\Theta}{ }^{2} \varepsilon^{-1 / 3} X$ with $C_{\Theta}=3.3$ (dashed lines) as predicted by Kolmogorov (1941). Lower panels show measured inner scale lo (solid lines) and that predicted by $7.4\left(v^{3} / \varepsilon\right)^{1 / 4}$ (dashed lines). 
Finally, we used the KH DNS to assess the correlation between TKE and CTKE as defined above for the turbulent component of the $\mathrm{KH}$ flow at various times. To separate turbulent from the larger scale, quasi-2-D KH motion, we computed TKE and CTKE at each location in the flow after a spanwise mean was removed. We then averaged the results streamwise and spanwise. The results of the correlation among these quantities are displayed in Figure 14 for six times throughout the active turbulence phase of the KH evolution. The result is an extremely high correlation as a function of depth for these
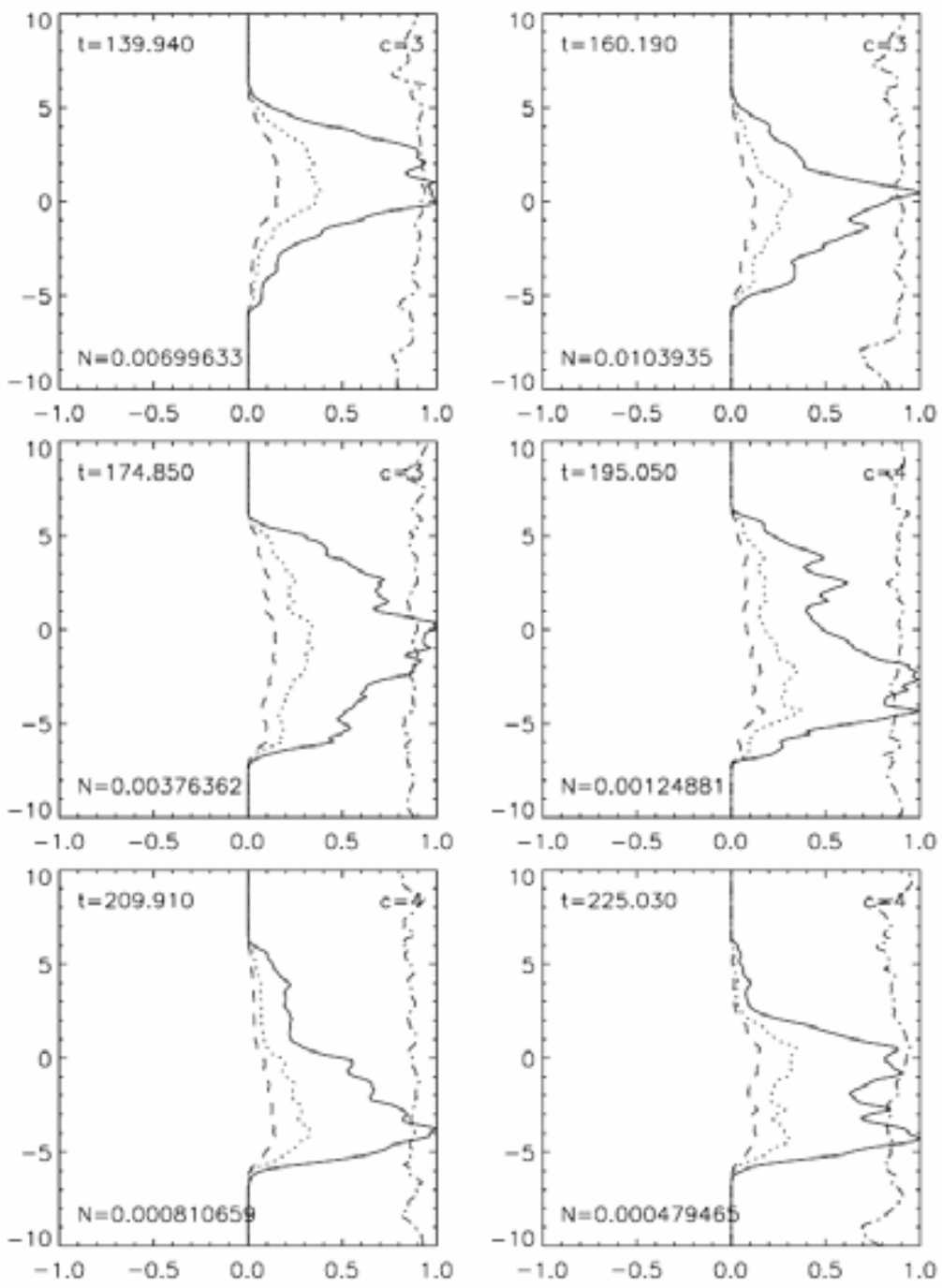

Figure 14. Streamwise and spanwise averages of $A^{2}=\left(u^{\prime 2}+v^{\prime 2}+w^{\prime 2}\right)^{2}$ (solid line), $B^{2}=$ $\left(\left(u^{\prime} v^{\prime}\right)^{2}+\left(u^{\prime} w^{\prime}\right)^{2}+\left(v^{\prime} w^{\prime}\right)^{2}\right)$ (dashed line), $A B$ (dotted line), and $A B /\left(A^{2}+B^{2}\right)^{1 / 2}$ (dash-dotted line) computed from the $\mathrm{KHI}$ data after removal of the spanwise average 2-D motion. Note the very high correlation between $\operatorname{CTKE}(A / 2)$ and $\operatorname{TKE}(B / 2)$ for all positions and times. As above, times are in $h / U_{0}$. 
averaged quantities. Thus, turbulence intensities are evaluated based on an average across a turbulence event, either TKE or CTKE would seem suitable for this purpose. However, such a correlation does not reflect the importance of coherent structures at larger scales, nor do the likely departures from the correlation of these averaged quantities locally within the flow.

\section{Impact of KHI Scales}

The implications of our data analysis and DNS findings are that the most intense vortices at several meter scales that are most likely to load and stress wind turbine blades and gearing most often accompany turbulence that arises from deep KHI, which arises from flows with significant wind shear (as opposed to small static stability). This is because large mean shears are required to seed instability and turbulence structures with large vorticity, and deep turbulence layers imply a shallower spectral slope, hence a more gradual decay in enstrophy to smaller specific scales of motion. If we take a vortex core of diameter $2 \mathrm{~m}$ as representative of those that torque turbine blades most strongly, these can arise from initial shear layer depths that range from $\sim 2 \mathrm{~m}$ upward. But a KHI at an initial shear layer depth of $20 \mathrm{~m}$ rather than $2 \mathrm{~m}$ (which implies a turbulence layer depth of $\sim 60 \mathrm{~m}$ rather than $6 \mathrm{~m}$ ) will impose a vorticity variance $\sim 10^{4 / 3} \sim 20$ times larger at $\sim 2-\mathrm{m}$ scales because the spectral character of the environment that gives rise to KHI has a spectral slope of order -3 , whereas that imposed by KHI-triggered turbulence has a slope nearer $-5 / 3$. The difference allows stronger vortices because the energy source is at larger scales.

\section{Recommendations for Implementing an Adverse Loading Risk Warning}

Characterizing boundary layer turbulence based only on $R i$ is not simple, as these estimates of flow stability or instability can be very misleading when flow gradients of temperature and wind are poorly resolved. And although CTKE (also TKE) and component vorticities generally correlated well, with turbulence episodes, neither was consistently large when turbulence, by other measures, was large (or small when other measures were small). Often, in fact, the component vorticities were large, because of significant gradients at small spatial scales, when the velocity variances were quite small. $C T K E$, on the other hand, did not falsely indicate significant turbulence because it is more sensitive to larger scale components of the motion spectrum.

\section{Conditions for Significant Turbulence}

Based on our analysis of Lamar data from September 2003 and the short interval recorded at the NWTC on 5 February 2001, we noted three conditions that were always met when turbulence was significant or severe:

- $R i$ tended to be small, typically less than 0.05 . 
- There was significant wind shear across the turbulence layer, typically $2-5 \mathrm{~m} / \mathrm{s}$, but occasionally much more ( $R i$ was small because of significant mean shear rather than because there was no stratification).

- Vertical velocities attained large values, typically a few $\mathrm{m} / \mathrm{s}$ or more, and exhibited coherent oscillations with periods of a few to tens of seconds.

When any of these conditions was not satisfied, there was no significant turbulence. The first condition was violated from $\sim 0600-0700$ UT on September 10 (where $R i>0.5$ ); it yielded a stable flow and little or no turbulence, despite persistent mean shears. Multiple cases of $R i<0$ were observed and discussed, but failed to have significant turbulence in the absence of mean shear. Finally, vertical velocities were always enhanced when significant turbulence was inferred based on any other measures.

\section{Recommended Measurements and Analysis}

Our observations appear to be quite robust, and suggest a clear strategy for measurements that would significantly mitigate the risk of adverse wind turbine loading by turbulence and coherent structures. Measurements of temperatures and vector winds at multiple levels are essential to computing stability, wind shear, $R i$, and vertical motions. But because the major adverse loading responses are likely due to larger scale structures, routine measurements probably do not need to be made with the same temporal resolution as for the CASES-99 and Lamar data sets. Measurements required to compute $R i$ having $0.2-1 \mathrm{~Hz}$ sensitivity (1-5 s temporal resolution) to horizontal winds and temperatures seem sufficient. To account for the relevant spatial structures under highwind $(\sim 20 \mathrm{~m} / \mathrm{s})$ conditions, however, measurements of vertical velocity at $\sim 5 \mathrm{~Hz}$ are probably necessary. Such measurements are especially important at the altitudes higher than $\sim 50 \mathrm{~m}$, where observations have indicated the largest responses.

Measurements that define mean wind shear, $R i$, and vertical velocity at $\sim 20 \mathrm{~m}$ vertical resolutions are probably sufficient to capture the larger scale and more energetic flow structures. Such measurements should likely also be performed upstream of the wind farms for general climatological boundary layer flows. Positioning measurements that surround a wind farm would afford even greater protection. A summary of these measurement recommendations follows.

- Temperature measurements at $0.2-1 \mathrm{~Hz}$ at $20-\mathrm{m}$ altitude intervals

- Horizontal wind measurements at $0.2-1 \mathrm{~Hz}$ at 20 -m altitude intervals

- Vertical wind measurements at $5 \mathrm{~Hz}$ and $20-\mathrm{m}$ altitude intervals.

These measurements upstream of the wind farm at enough sites provide sensitivity to most climatological flow conditions.

Such measurements at coarser altitude resolution would also provide significant protection. However, the major costs of such a warning system would likely be the 
installation, maintenance, and data processing, which would not be greatly affected by the recommended higher measurement resolution.

Analysis of the results that report wind shear (vertical shear of the horizontal wind) and $R i$ at 0.2 to $1 \mathrm{~Hz}$ and vertical velocities at $5 \mathrm{~Hz}$ would enable turbulence risk to be assessed and reported. Based on the analysis, suggestions of thresholds for significant risk (which would likely require tuning based on correlations of these quantities and measured wind turbine loading, turbine type, altitude, etc.) include all of the following:

- At least one measurement of $R i<0.05$ for 5 -s average within the previous $30 \mathrm{~s}$ (a 5-s average is likely better than a 1-s average for $R i$ assessment)

- Mean wind shear that exceeds $2 \mathrm{~m} / \mathrm{s}$ across the measured altitude interval (a smaller mean wind shear is unlikely to enable structures that result in vertical velocities of $\mathrm{w}$ $\sim 2 \mathrm{~m} / \mathrm{s}$ or greater)

- Vertical velocities (at $5 \mathrm{~Hz}$ ) that exceed either an RMS of $1 \mathrm{~m} / \mathrm{s}$ or multiple values that exceed $2 \mathrm{~m} / \mathrm{s}$ for a 5 -s interval.

\section{Predicting Boundary Layer Flows with Mesoscale Models}

Accurate and reliable predictions of boundary layer structure and variability on the spatial and temporal scales pose large problems because the external boundary conditions necessary for local high-resolution forecasting at these scales is generally not available. Nor are mesoscale models sufficiently advanced to describe boundary layer flows at scales of tens of meters in altitude. However, such modeling would be possible with good external data constraints and inputs, and sufficient vertical resolution. The goal would not be to describe the wave and instability structures that lead to enhanced turbulence, as this remains far beyond the scope of current modeling (and input data specification) capabilities. But with good upstream characterization of the boundary layer structure and stability (particularly mean wind shear, temperature gradients, and $R i$ ), forecasting the evolution of this state, and of the related potential for significant instability events, seems possible. Such a forecasting capability would likely represent a significant development effort at this stage, as these needs are considerably beyond the accomplishments of such models. Such a capability would probably also need to be supported by its own local data network to provide upstream information and external constraints. 


\section{References}

Bolgiano, R., 1959. "Turbulent Spectra in a Stably Stratified Atmosphere," J. Geophys. Res. 64:2226-2229.

Fritts, D.C. and J.A. Werne, 2000. "Turbulence Dynamics Due to Gravity Waves in the Lower and Middle Atmosphere," AGU Monograph, Atmospheric Science across the Stratopause, pp. 143-159.

Kelley, N., M. Hand, S. Larwood, and E. McKenna, 2002. "The NREL Large-Scale Turbine Inflow and Response Experiment - Preliminary Results," NREL/CP-500-30917; National Renewable Energy Laboratory, Golden, CO.

Kolmogorov, A.N., 1941. "Turbulence and Stochastic Processes: Kolmogorov's Ideas 50 Year On, eds. J.C.R. Hunt, O.M. Phillips, and D. Williams, Proc. R. Soc. London, Ser. A 434, 1991. Pages 9-13, "The Local Structure of Turbulence in Incompressible Viscous Fluid for Very Large Reynolds Numbers," Dokl. Akad. Nauk. SSSR 30, 4, 1941. Pages 15-17, "Dissipation of Energy in the Locally Isotropic Turbulence," Dokl. Akad. Nauk. SSSR 32, 1, 1941.

Poulos, G. S., W. Blumen, D. C. Fritts, J. K. Lundquist, J. Sun, S. P. Burns, C. Nappo, R. Banta, R. Newsom, J. Cuxart, E. Terradellas, B. Balsley and M. Jensen, 2002: CASES99: A Comprehensive Investigation of the Stable Nocturnal Boundary Layer, Bull. Amer. Meteor. Soc., 83, 555-581.

Werne, J.A., C. Bizon, and D.C. Fritts, 2001. "Wave Breaking and Shear Turbulence Simulations in Support of the Airborne Laser," 11th DoD HPC User Group Symposium, Biloxi, MS.

Werne, J.A. and D.C. Fritts, 1998. "Turbulence in Stratified and Sheared Fluids: T3E Simulations," 8th DoD HPC User Group Symposium, Redondo Beach, CA.

Werne, J.A. and D.C. Fritts, 1999a. "Stratified Shear Turbulence: Evolution and Statistics," Geophys. Res. Lett. 26:439-442.

Werne, J.A. and D.C. Fritts, 1999b. "Anisotropy in Stratified Shear Turbulence," 9th DoD HPC User Group Symposium, Monterey, CA.

Werne, J.A. and D.C. Fritts, 2000. "Structure Functions in Stratified Shear Turbulence," 10th DoD HPC User Group Symposium, Albuquerque, NM.

Werne, J.A. and D.C. Fritts, 2001. "Anisotropy in a Stratified Shear Layer," Phys. Chem. Earth 26:263-268. 


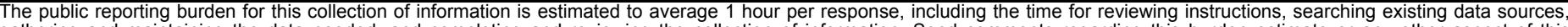

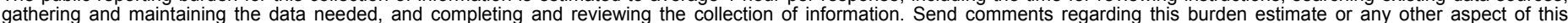

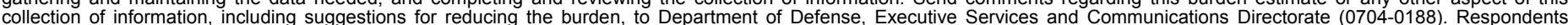

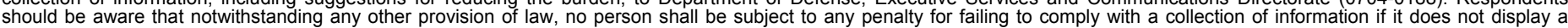

should be aware that notwithstand

PLEASE DO NOT RETURN YOUR FORM TO THE ABOVE ORGANIZATION.

\section{REPORT DATE (DD-MM-YYYY) July 2005 \\ 2. REPORT TYPE \\ Subcontractor Report}

4. TITLE AND SUBTITLE

Conditions That Contribute to Adverse Wind Turbine Loading in the Nocturnal Boundary Layer: Final Report

ing

3. DATES COVERED (From - To)

11/15/2003 - 12/31/2004

5a. CONTRACT NUMBER

DE-AC36-99-GO10337

5b. GRANT NUMBER

5c. PROGRAM ELEMENT NUMBER

5d. PROJECT NUMBER

NREL/SR-500-37809

5e. TASK NUMBER

WER4-3002

5f. WORK UNIT NUMBER
7. PERFORMING ORGANIZATION NAME(S) AND ADDRESS(ES)

Colorado Research Associates

3380 Mitchell Lane

Boulder, CO 80301

9. SPONSORING/MONITORING AGENCY NAME(S) AND ADDRESS(ES)

National Renewable Energy Laboratory

1617 Cole Blvd.

Golden, CO 80401-3393

\section{PERFORMING ORGANIZATION REPORT NUMBER}

10. SPONSOR/MONITOR'S ACRONYM(S) NREL

11. SPONSORING/MONITORING AGENCY REPORT NUMBER NREL/SR-500-37809

12. DISTRIBUTION AVAILABILITY STATEMENT

National Technical Information Service

U.S. Department of Commerce

5285 Port Royal Road

Springfield, VA 22161

13. SUPPLEMENTARY NOTES

NREL Technical Monitor: Neil Kelley

14. ABSTRACT (Maximum 200 Words)

This report summarizes the development of a methodology to describe the characteristics of coherent turbulence in the nocturnal boundary layer that are known to induce excessive structural loads and component vibration in operating wind turbines and suggestions for applying those results in the development of techniques of real-time detection and prediction that can be used for mitagation purposes.

\section{SUBJECT TERMS}

Wind turbines; coherent turbulence loading; nocturnal boundary layer; Kelvin-Helmholtz Instability; adverse turbulent conditions prediction

\begin{tabular}{|c|c|c|c|c|}
\hline \multicolumn{3}{|c|}{ 16. SECURITY CLASSIFICATION OF: } & \multirow{2}{*}{$\begin{array}{l}\text { 17. LIMITATION } \\
\text { OF ABSTRACT } \\
\text { UL }\end{array}$} & \multirow{2}{*}{$\begin{array}{l}\text { 18. NUMBER } \\
\text { OF PAGES }\end{array}$} \\
\hline $\begin{array}{l}\text { a. REPORT } \\
\text { Unclassified }\end{array}$ & $\begin{array}{l}\text { b. ABSTRACT } \\
\text { Unclassified }\end{array}$ & $\begin{array}{l}\text { c. THIS PAGE } \\
\text { Unclassified }\end{array}$ & & \\
\hline
\end{tabular}

19a. NAME OF RESPONSIBLE PERSON

19b. TELEPHONE NUMBER (Include area code) 